\title{
Color Blobs in Cortical Areas V1 and V2 of the New World Monkey Callithrix jacchus, Revealed by Non-Differential Optical Imaging
}

\author{
Matthias F. Valverde Salzmann, ${ }^{1}$ Andreas Bartels, ${ }^{1,2}$ Nikos K. Logothetis, ${ }^{1,3}$ and Almut Schüz ${ }^{1}$ \\ ${ }^{1}$ Max Planck Institute for Biological Cybernetics, D-72076 Tübingen, Germany, ${ }^{2}$ Vision and Cognition Laboratory, Centre for Integrative Neuroscience, \\ University of Tübingen, D-72076 Tübingen, Germany, and ${ }^{3}$ Division of Imaging Science and Biomedical Engineering, University of Manchester, \\ Manchester M13 9PT, United Kingdom
}

Color vision is reserved to only few mammals, such as Old World monkeys and humans. Most Old World monkeys are trichromats. Among them, macaques were shown to exhibit functional domains of color-selectivity, in areas V1 and V2 of the visual cortex. Such color domains have not yet been shown in New World monkeys. In marmosets a sex-linked dichotomy results in dichromatic and trichromatic genotypes, rendering most male marmosets color-blind. Here we used trichromatic female marmosets to examine the intrinsic signal response in V1 and V2 to chromatic and achromatic stimuli, using optical imaging. To activate the subsystems individually, we used spatially homogeneous isoluminant color opponent (red/green, blue/yellow) and hue versus achromatic flicker (red/gray, green/gray, blue/gray, yellow/gray), as well as achromatic luminance flicker. In contrast to previous optical imaging studies in marmosets, we find clearly segregated color domains, similar to those seen in macaques. Red/green and red/gray flicker were found to be the appropriate stimulus for revealing color domains in single-condition maps. Blue/gray and blue/yellow flicker stimuli resulted in faint patch-patterns. A recently described multimodal vessel mapping approach allowed for an accurate alignment of the functional and anatomical datasets. Color domains were tightly colocalized with cytochrome oxidase blobs in V1 and with thin stripes in V2. Thus, our findings are in accord with 2-Deoxy-D-glucose studies performed in V1 of macaques and studies on color representation in V2. Our results suggest a similar organization of early cortical color processing in trichromats of both Old World and New World monkeys.

\section{Introduction}

Color vision in primates is segregated into the parvocellular (PC) [red/green ( $\mathrm{L}-\mathrm{M})$-cone)], the koniocellular (KC) [blue/yellow (S-(L+M)-cones)], and the magnocellular (MC) pathway [luminance $(\mathrm{L}+\mathrm{M})$-cones)]. These pathways originate in the retina and project in parallel to V1 via the LGN (Casagrande, 1994).

Anatomical investigations in macaques and owl monkeys showed that cytochrome oxidase (CO) blobs (Wong-Riley, 1979; Horton and Hubel, 1981) receive inputs from the KC layers of the LGN (Hendry and Yoshioka, 1994; Ding and Casagrande, 1997, 1998). Reports on PC and MC inputs to V1 are contradictory, but some find preferences for $\mathrm{PC}$ projections to blobs and MC projections to interblobs (via layer 4C $\alpha$ ) (Sincich and Horton, 2005).

\footnotetext{
Received Sept. 22, 2011; revised Feb. 15, 2012; accepted March 29, 2012

Author contributions: M.F.V.S. designed research; M.F.V.S. performed research; M.F.V.S., A.B., and N.K.L. contributed unpublished reagents/analytic tools; M.F.V.S. and A.B. analyzed data; M.F.V.S., A.B., and A.S. wrote the paper.

This work was supported by the Max Planck Society. We are grateful to Adrian Streit (Max Planck Institute for Developmental Biology, Tübingen, Germany) for help and support on the genetic profiling of our animals. Special thanks go to Anna Wang Roe and Damian Wallace for helpful discussions on optical imaging in marmosets. We are grateful to Jan Kremers for discussions on the visual system of marmosets and to Frank Schaeffel for support on marmoset refraction.

Correspondence should be addressed to Matthias F. Valverde Salzmann, Department of Physiology of Cognitive Processes. Max Planck Institute for Biological Cybernetics, Spemannstrasse 38, D-72076 Tübingen, Germany. E-mail: matthias.valverde@tuebingen.mpg.de.

DOI:10.1523/JNEUROSCI.4832-11.2012

Copyright $\odot 2012$ the authors $\quad 0270-6474 / 12 / 327881-14 \$ 15.00 / 0$
}

Thus, a blob-related segregation of V1 by geniculate inputs seems to be in accordance with early findings of Livingstone and Hubel (1984), suggesting that blobs are color processing domains. However, this functional-anatomical relation is highly controversial (Martin, 2004).

Tootell et al. (1988) investigated cortical responses to color opponent and color/achromatic flicker stimuli by 2-deoxy-Dglucose (2DG) autoradiography in macaques. They showed a colocalization of $\mathrm{CO}$ blobs with regions of increased 2DG labeling. These findings were supported by optical imaging (OI) studies in macaques (Landisman and Ts'o, 2002; Xiao et al., 2007; Lu and Roe, 2008). There, color domains were mapped by differential OI: single-condition maps evoked by color opponent gratings were correlated with those evoked by achromatic gratings. Notably, Landisman and Ts'o (2002) found that red/green but not blue/yellow gratings allowed reliable blob imaging, compromising their initial assumption that S-cone-selective stimuli would allow them to investigate the segregation of PC and KC inputs.

A polymorphism that occurs in marmosets and other New World monkeys could play a key role in investigations of color vision. The marmoset $\mathrm{X}$-chromosome carries only one gene coding for one of three (L-M)-cones (Williams et al., 1992; Jacobs et al., 1993; Yeh et al., 1995; Kawamura et al., 2001). Two of them each coding for a slightly different L- or M-cone are necessary for trichromatic vision. Thus, most males and some females show 
dichromatism (Hunt et al., 1993), which impairs their ability to distinguish reds and greens (Blessing et al., 2004; Conway, 2009).

Two studies investigated whether dichromatic marmosets expose color domains, one using differential OI (red/green versus blue/yellow gratings) (Roe et al., 2005b), the other one using single-condition OI (red/green, blue/yellow gratings) (Buzás et al., 2008). Functional domains were not found, suggesting a homogeneous distribution of KC inputs into V1.

Here we studied trichromatic marmosets by using singlecondition OI. We found that color opponent flicker revealed functional color domains in these animals. Alignments with anatomical stains revealed a colocalization with $\mathrm{CO}$ blobs in $\mathrm{V} 1$ and with thin stripes in V2. These results resemble those shown for macaques (Michael, 1981; Livingstone and Hubel, 1984; Yoshioka and Dow, 1996; Roe and Ts'o, 1999; Ts'o et al., 2001; Landisman and Ts'o, 2002; Xiao et al., 2003, 2007; Lu and Roe, 2008; Lim et al., 2009) and suggest a similar organization of early cortical color processing in trichromatic Old World and New World monkeys.

\section{Materials and Methods}

Animals. Three female adult marmosets (Callithrix jacchus; $400-550 \mathrm{~g}$ ) were used in this study (see also Valverde Salzmann et al., 2011).The animals were kept in pairs in enriched climatized cages of up to $6 \mathrm{~m}^{3}$ in size, and received water ad libitum and daily fresh and changing fruits, vegetables, Arabic gum (Marmoset gum, Mazuri Europe), marmoset pellets (Mini Marex, Mazuri Europe), and animal proteins in the form of eggs, locusts, and mealworms. All surgical and experimental procedures were approved by the local authorities (Regierungspraesidium) and are in full compliance with the directive of the European community for the protection of animals used for experimental and other scientific purposes (86/609/EEC). For a detailed description of our surgical, anesthesia protocols and of the alignment of anatomical and functional data, see Valverde Salzmann et al. (2011).

Surgery, anesthesia. Animals were anesthetized using remifentanil (Ultiva, GlaxoSmithKline; $0.06-0.1 \mu \mathrm{g} / \mathrm{kg} / \mathrm{min}$, i.v.) and additional doses of sevofluran (Sevorane, Abbott Laboratories) (4-6\% in medical $\mathrm{O}_{2}$ applied during the surgery via a facemask and $0-1.5 \%$ during experimental conditions via tracheal tube). Muscle relaxation during the experiment was achieved using Mivacron $(1 \mathrm{mg} / \mathrm{kg}$ body weight/h, i.v.). All incision sites were additionally pretreated with local injections of Lidocain- $\mathrm{HCl}(2 \%, \mathrm{~B}$. Braun). The femoral vein was cannulated for the infusion of the opioids and additional electrolyte solutions (Amynin, Merial; Jonosteril, Fresenius Kabi). A tracheotomy was performed for mechanical ventilation. Animals were respirated at 38-45 strokes/min with a mixture of medical air and $\mathrm{O}_{2}$ (ratio 80\%:20\%). Endtidal $\mathrm{CO}_{2}$ was measured and kept at $3.4 \pm 1 \%$ by adjusting respiration rate and/or stroke volume. A craniotomy of $\sim 5 \mathrm{~mm}$ radius was made over the visual cortex. The dura mater was kept intact. An optical chamber was implanted and filled with agarose gel $(6 \%$ in $\mathrm{NaCl}$, type VII Sigma-Aldrich Chemie). Physiological parameters and anesthetic depth were constantly monitored by recording ECG, EEG, $\mathrm{SpO}_{2}$ (kept at $>98 \%$ ), and body temperature. The latter was adjusted to $37 \pm 1{ }^{\circ} \mathrm{C}$ via a heated silicone gel pad.

Preparation for optical recordings. The animals were mounted into a stereotactic apparatus and the eyes were focused on the stimulus screen (24 inch LCD monitor, gamma corrected, Belineao.display 4.1, Belinea; refresh rate, $70 \mathrm{~Hz}$ )—placed $50 \mathrm{~cm}$ in front of the animals' eyes-by
Table 1. Stimulus specifications: CIE coordinates for all hues of the color-varying and luminance-varying stimuli

\begin{tabular}{lcll}
\hline Stimulus hues & $\begin{array}{l}\text { Luminance } \\
\left(Y: c \mathrm{~d} / \mathrm{m}^{2}\right)\end{array}$ & & \multicolumn{2}{l}{ (IE coordinates } \\
\cline { 4 - 4 } Gray (blank) & 80.57 & 0.3062 & $y$ \\
Red & 39.6 & 0.6471 & 0.3165 \\
Gray & 37.54 & 0.3043 & 0.3382 \\
Red & 39.31 & 0.6465 & 0.3139 \\
Green & 36.7 & 0.2733 & 0.3378 \\
White & 179.23 & 0.309 & 0.59 \\
Black & 0.2 & 0.259 & 0.3333 \\
Blue & 13.29 & 0.1445 & 0.2567 \\
Gray & 9.74 & 0.3028 & 0.0642 \\
Blue & 13.21 & 0.1445 & 0.3119 \\
Yellow & 12.32 & 0.4075 & 0.0641 \\
Green & 35.49 & 0.2734 & 0.4881 \\
Gray & 35.74 & 0.3047 & 0.5903 \\
Yellow & 163.11 & 0.4152 & 0.3149 \\
Gray & 166.22 & 0.3114 & 0.5093 \\
\hline
\end{tabular}

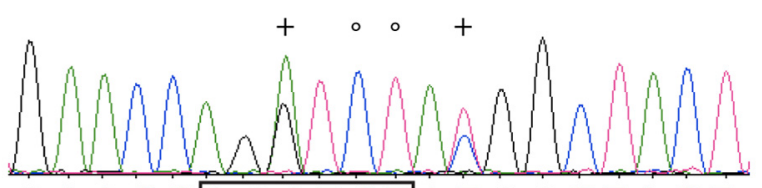

exon2-543nm: G A A C C A G A T C TA T G G C T A C T exon2-556nm: G A A C C A G G T C T A C G G C T A C T exon2-563nm: G A A C C A G G T T C A C G G C T A C T

exon5-543nm: GCA G A C G C A G T A C G C C A C G A exon5-556nm: GCA G A $\subset A \subset A G T A C G \subset C G \subset G A$ exon5-563nm: $G \subset A G A \subset G \subset A A T A \subset G \subset \subset A \subset G A$

Figure 1. Genotype determination. A shows the sequences of the three alleles around the $543 \mathrm{~nm}$-specific Bglll site (boxed) in xon 2 described by Blessing et al. (2004). The sequence shown is derived from M3. The sense strand is shown. The other two a dhe three alleles around the $556 \mathrm{~nm}$-specific $\mathrm{Apy}$ CH4lll site (boxed) in exon 5

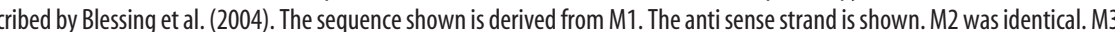
列 AF051595 (exon 2-543 nm), AY142349 (exon 5-543 nm), AF051589 (exon 2-556 nm), AF227248 (exon 2-556 nm), XM_002763410 (whole gene $563 \mathrm{~nm}$ ).

using PMM contact lenses (Soflex Israelens Contact Lenses Ltd.) and an infrared video refractor (Schaeffel et al., 1994; Troilo et al., 2007). The stimulus screen spanned $55^{\circ}$ horizontal $\times 37.5^{\circ}$ vertical of the animal's central field of view. The eyes were moistened with saline in regular intervals and a clear view through the artificial lenses was ensured throughout experimental conditions by periodic eye checks.

Optical imaging. We recorded the intrinsic signals at red light illumination (6xLED ring light, peak wavelength $634 \mathrm{~nm}$ Nichia $5 \mathrm{~mm}$ LED NSPR510CS, Lumitronix LED-Technik) and an Optical Imaging Inc. 3001 system with a Dalsa 1M60P camera. Reflectance changes were recorded at $60 \mathrm{~Hz}$ at full camera resolution $(1020 \times 1020$ pixel $)$. Temporal binning was applied, resulting in a reduction of the raw frame rate to 5 $\mathrm{Hz}$. All stimuli were presented randomly 40 times in blocks. Each block consisted of a set of 2, 6 or 7 stimuli and a blank stimulus (see Visual stimuli for details). During the blank condition, as well as the interstimulus phases, a gray screen was presented to the animal. Stimuli were presented for 3-4 s, $2 \mathrm{~s}$ after frame recording started. Fifty frames were recorded during each presentation. Interstimulus intervals ranged be- 
tween 13 and $15 \mathrm{~s}$. The cortical responses to each stimulus were averaged. Thus, each stimulus condition showed the time course of the mean reflectance changes of 40 stimulations over 50 frames.

Visual stimuli. All stimuli were generated by an OpenGL custom program (Sheinberg and Logothetis, 2001) (see Preparation for optical recordings for monitor description). We used four classes of stimuli. In two of them, one uniform full-screen color was alternated with another uniform full-screen color in time at $1.5 \mathrm{~Hz}$ (temporal profile: square wave). Using the terminology of Tootell et al. (1988), we refer to these as colorvarying stimuli, where one hue was alternated with another, isoluminant hue, with pairs being red/green, blue/yellow, red/gray, green/gray, blue/ gray, and yellow/gray. The second class were luminance-varying stimuli, where black and white were alternated, and the third was a blank condition (gray screen). A final set of stimuli consisted of achromatic moving square wave gratings (spatial frequency 1.2 cycles/degree; temporal frequency $2 \mathrm{~Hz}$ ) of six different orientations that alternated movement direction every $0.75 \mathrm{~s}$. The luminance $\left(\mathrm{cd} / \mathrm{m}^{2}\right)$ and spectral coordinates $(x, y)$ of each stimulus color were measured using a Konica Minolta CS1000 spectrometer, and are listed in Table 1. Table 3 lists the estimated marmoset-specific L,M,S-cone activations elicited by each of the stimuli (see Results).

Three stimulus block protocols (a to c) were used in this study. The first stimulus block (a) consisted of one color-varying condition (e.g., red/green) and a luminance flicker condition (i.e., black/white) as well as a blank condition (gray screen). This protocol was applied only in case M1. The second stimulus block (b) consisted of all color-varying conditions, the luminance-varying condition, and the blank condition. This protocol was used to stimulate cases M2 and M3. The third stimulus block (c) used achromatic drifting square wave gratings and was presented to each animal after the color stimulus blocks. All stimuli were presented binocularly.

In addition, two animals were stimulated monocularly. For this purpose the eye ipsilateral to the cortical hemisphere on which imaging was performed was covered with black tape. Animal M1 viewed the stimulus protocols $(\mathrm{a}, \mathrm{c})$ and animal M2 viewed protocols $(\mathrm{b}, \mathrm{c})$ through their contralateral eyes.

Intrinsic optical imaging data processing. Intrinsic optical imaging (IOI) data processing was performed using custom written scripts executed in Matlab (The MathWorks). Image processing was done pixelwise in all cases. Map calculations and filtering were based on a modified version (Valverde Salzmann et al., 2011) of protocols published by Lu and Roe (2007). We used a frame-zero-subtraction to calculate single-condition map time series of the optical reflectance changes $(\Delta R / R)$ for the relevant 46 (of 50) frames of each stimulus condition. The map of the singlecondition map series that revealed the response pattern most clearly was used for further signal correlation analysis. This was usually a map corresponding to the point in time $1-2 \mathrm{~s}$ after stimulus onset. In single conditions where no pattern was observed, the map corresponding in time to the pattern map was used for further demonstration. To increase the contrast of the single-condition maps we set a threshold level of $\pm 5 \%$ for clipping the pixel intensities. Only maps of case M3 were clipped at lower levels $( \pm 2 \%)$ for enhanced visualization of functional compartmentalization. The maps of case M3 in Figure 6 (see below) were also clipped at $\pm 5 \%$. Contours of signal compartments were calculated using the contour method (Matlab). To determine the center coordinates of color patches, we refer to a method by Lu and Roe (2008). Discs with a diameter of $200 \mu \mathrm{m}$ were fitted to the position of signal patches. A distance measurement of the disc center coordinates was then performed based on the location of nearest neighbors (Landisman and Ts'o, 2002).

Histology. At the end of the experiment the anesthetic depth was increased by adjusting the rate of sevofluran to $5 \%$ and the remifentanil infusion was increased to $0.5 \mu \mathrm{g} \cdot \mathrm{kg}^{-1} \cdot \mathrm{min}^{-1}$. Animals were then killed by an injection of a lethal dose of pentobarbital ( $1.5 \mathrm{ml}$ of Narcoren, $160 \mathrm{mg} / \mathrm{ml}$, intracardially). The animal was perfused through the heart with $100-150 \mathrm{ml}$ of $\mathrm{PB}(0.1 \mathrm{M})$ followed by $300 \mathrm{ml}$ of $4 \%$ buffered PFA (Roti-Histofix, Carl Roth $\mathrm{GmbH}$ ). After that, animals were processed for labeling of the brain vasculature by perfusion with fluorescence-labeled gelatin. Before cutting tangential sections with a freezing microtome, we took an image of the labeled surface vasculature

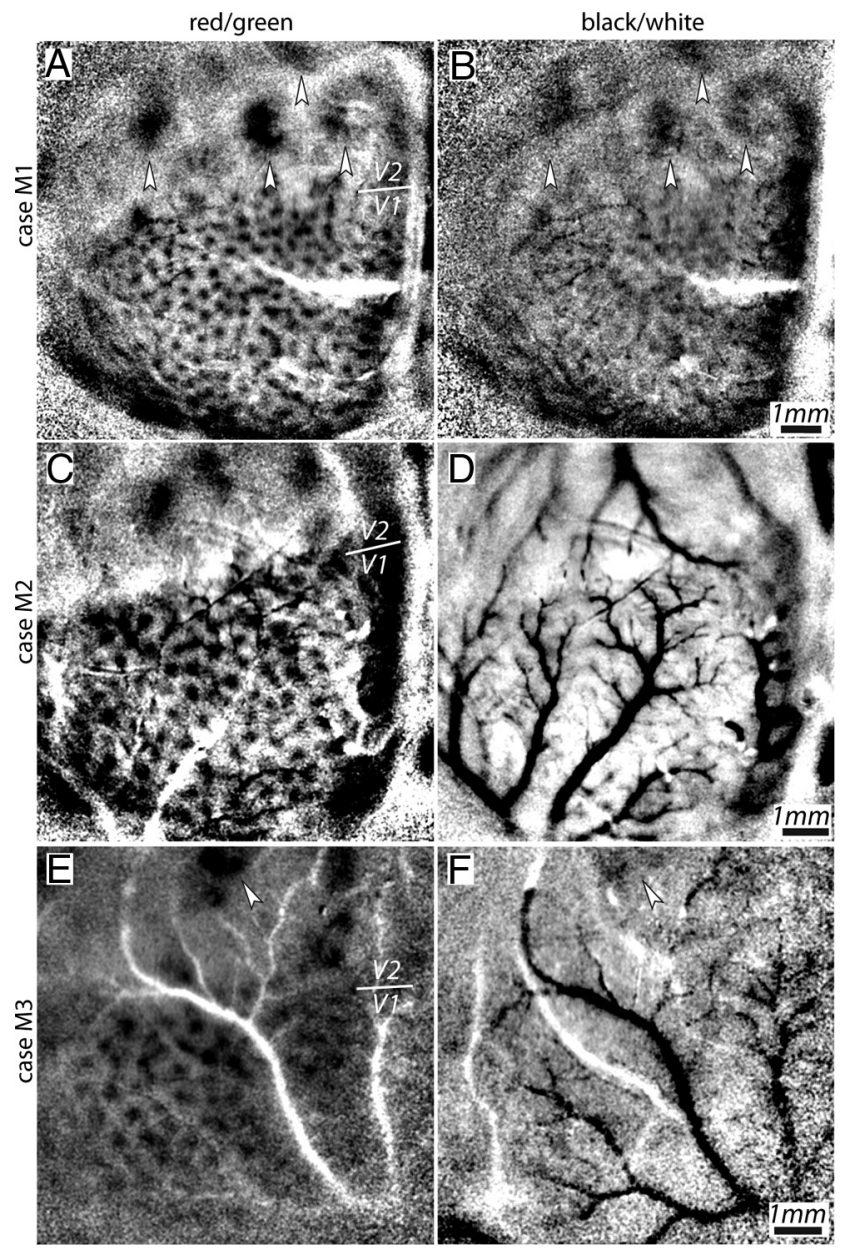

Figure 2. Functional color patches in the visual cortex of a marmoset. $\boldsymbol{A}-\boldsymbol{F}$, single-condition maps from intrinsic 0 r recorded in the left hemisphere of 3 marmosets (superior sagittal sinus is located on the right side of the pictures). $\boldsymbol{A}, \boldsymbol{C}$, and $\boldsymbol{E}$ show $10 \mathrm{I}$ maps of the intrinsic response to color-varying (red/green) flicker stimuli. A patchy pattern of activated functional domains can be seen. In V1 (lower half of the pictures) the mapped domains show the typical distance of $400-450 \mu \mathrm{m}$ (see Colocalization of functional color patches with CO blobs in visual area V1 for quantification). In V2 (upper half of the pictures) larger areas with center to center distances of $2-2.5 \mathrm{~mm}$ responded to the stimulus. $\boldsymbol{B}, \boldsymbol{D}$, and $\boldsymbol{F}$ show the corresponding intrinsic response maps obtained with luminance-varying (black/white). These maps lack any clear pattern formation.

of the imaged cortex region. This image served as a template of the physiological state of the cortex used for the precise alignment of the histological preparations to the functional IOI maps. A modified version of the CO staining protocol by Wong-Riley (1979) was used to reduce distortions (see Valverde Salzmann et al., 2011, for modifications) during tissue processing. Images of the stained sections were taken using an AxioImager.Z1 microscope (Zeiss) with a $5 \times$ objective (Neofluar, Zeiss). The images were then registered and aligned to the functional data, and a pseudo three-dimensional reconstruction of the sectioned tissue was made to take into account the three-dimensional nature of the cortical architecture, particularly with regard to cortical curvature and the tilt of radial structures, such as vessels and cortical columns (Valverde Salzmann et al., 2011).

Histological image processing. The images of the stained sections were filtered in the spatial domain by using a Gaussian $(\sigma=35 \mu \mathrm{m})$ and a radial filter (diameter $=400 \mu \mathrm{m}$ ). The images of 3 adjacent sections exhibiting blob patterns of V1 were averaged to increase signal-to-noise ratio. The same was done on the images of the three sections exhibiting the stripes of V2. In doing so, we also took the depth shift of the CO signal due to cortical curvature into account. This approach was derived from the multimodal vessel mapping approach used here in combination with 
the three-dimensional reconstruction of the processed cortical tissue (Valverde Salzmann et al., 2011). For a center distance measurement of CO blobs, the same method as described in IOI data processing for color patch distance measurements was applied.

Genotype determination. Samples for the genetic analysis were taken from the bloodless liver of the perfused animals to avoid falsepositive results due to chimerism (Benirschke et al., 1962; Signer et al., 2000; Kawamura et al., 2001). The tissue samples were washed 6 times for $10 \mathrm{~min}$ in $1 \mathrm{ml}$ of TE $(10 \mathrm{~mm}$ Tris, $1 \mathrm{~mm}$ EDTA, $\mathrm{pH} 8.0$ ) at $55^{\circ} \mathrm{C}$. Then the samples were incubated in $50 \mu \mathrm{l}$ of TE supplemented with $1.7 \mathrm{mg} / \mathrm{ml}$ proteinase $\mathrm{K}$ for $2 \mathrm{~h}$ at $55^{\circ} \mathrm{C}$. TE $(100$ $\mu l)$ supplemented with $1 \mathrm{mg} / \mathrm{ml}$ proteinase $\mathrm{K}$ was added to each sample and incubation continued at $55^{\circ} \mathrm{C}$ for $3 \mathrm{~h}$ followed by $10 \mathrm{~min}$ incubation at $75^{\circ} \mathrm{C}$ to inactivate the proteinase $\mathrm{K}$. One microliter of the solution per reaction was used as template for PCR amplification of exons 2 and 5 of the $\mathrm{X}$ chromosomal rod opsin gene using the primers described previously (Hunt et al., 1993; Yeh et al., 1995; Blessing et al., 2004). The PCR was performed in $25 \mu$ l of PCR buffer (New England Biolabs) in the presence of $0.2 \mathrm{~mm}$ each primer, $0.04 \mathrm{~mm}$ each dNTP, $0.2 \mathrm{mg} / \mathrm{ml} \mathrm{BSA}$ and $1.5 \mathrm{U}$ of TaqDNA Polymerase (New England Biolabs). For the PCR program, an initial denaturation step $\left(94^{\circ} \mathrm{C}\right.$ for $\left.2 \mathrm{~min}\right)$ was followed by 35 cycles of $94^{\circ} \mathrm{C}$ for $30 \mathrm{~s}$ (denaturing), $57^{\circ} \mathrm{C}$ for $30 \mathrm{~s} \mathrm{(an-}$ nealing), $72^{\circ} \mathrm{C}$ for $30 \mathrm{~s}$ (extension), and a final extension step $\left(72^{\circ} \mathrm{C}\right.$ for $\left.7 \mathrm{~min}\right)$. The resulting PCR products were agarose gel purified using the QIAquick gel extraction kit (Qiagen) and submitted to the on campus sequencing facility at the Max Planck Institute for Developmental Biology for sequencing from both sides using the same primers as for the PCR. The sequences were compared with the published sequences for the different X chromosomal rod opsin alleles (GenBank accession numbers: AF051595, exon 2,543 $\mathrm{nm}$; AY142349, exon 5, $543 \mathrm{~nm}$; AF051589, exon 2, 556 nm; AF227248, exon $2556 \mathrm{~nm}$; XM_002763410, whole gene, $563 \mathrm{~nm}$ ). See Figure 1 for exemplary demonstration of the sequencing results of exon 2 and 5.

\section{Results}

Color domains in marmosets

In each of the three monkeys (M1, M2, M3) used for this study, visual stimulation with red/green flicker of a low temporal frequency of $1.5 \mathrm{~Hz}$ resulted in intrinsic signal maps showing a patchy columnarlike distribution of signal peaks in primary and secondary visual areas (Fig. $2 A, C, E$ ). The patches were clearly segregated from neighboring patches by zones of low intrinsic signal responses indicating low stimulus specificity. Thus, these patterns showed the typical appearance of functional color domain patterns reported in previous studies in the macaque monkey (Lu and Roe, 2008). In contrast, black/white flicker presented to the same animals resulted in maps without obvious pattern formation (Fig. $2 B, D, F$ ).
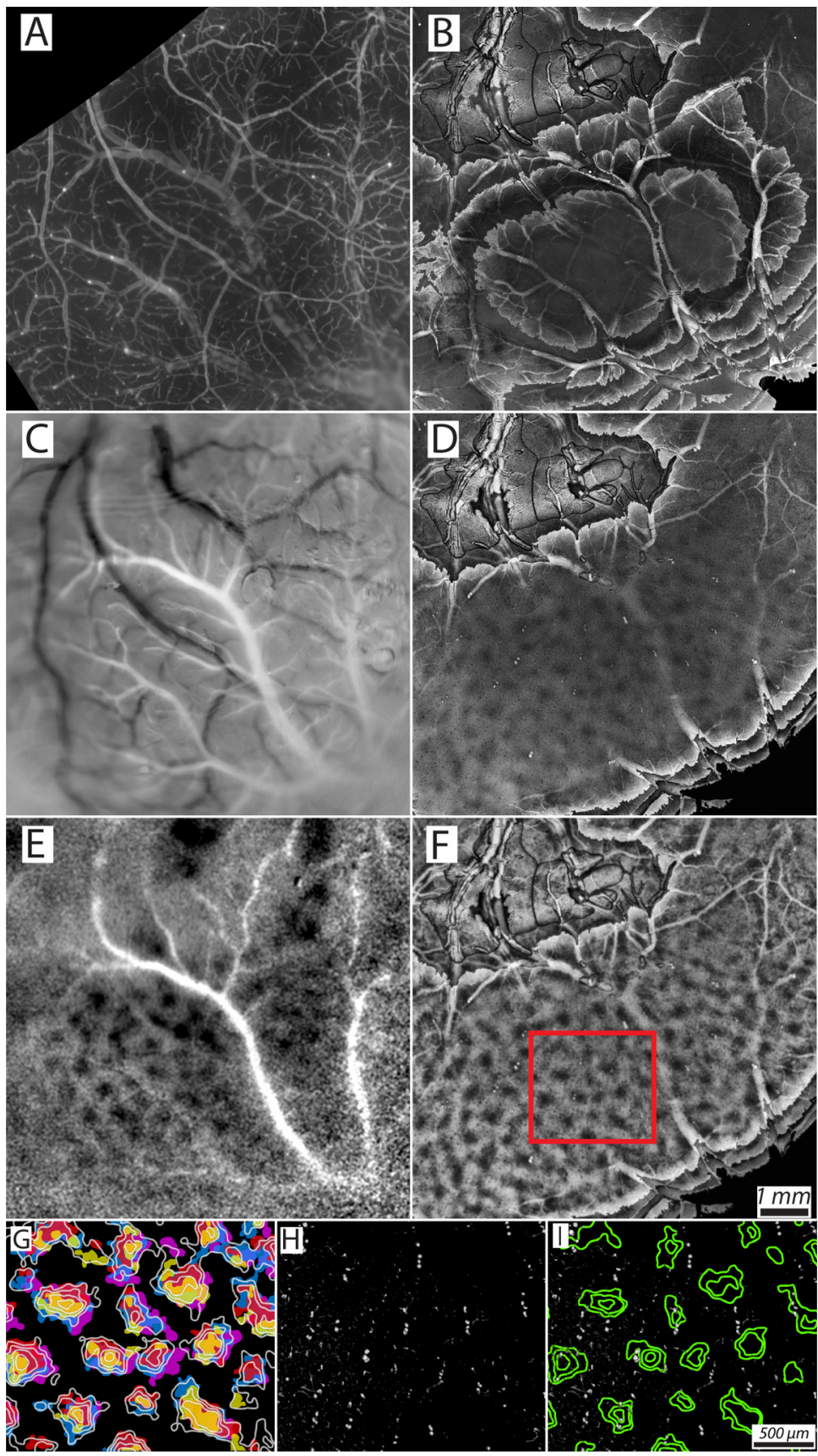

Figure 3. Alignment of functional domains to CO rich zones in histological sections. A multimodal vessel mapping approach was applied to ensure accurate alignment (Valverde Salzmann et al., 2011). A, FITC-vessel-map of the cortical surface vasculature. B, A stack of tangential sections through the visual cortex, registered to the FITC vessel map. $C$, IOI-vessel-map that was used to align the FITC vessel map in $\boldsymbol{A}$, as well as the 3 D reconstruction in $\boldsymbol{B}$, to the functional data. $\boldsymbol{D}$, Section from lower layer $2 / 3$ through $V 1$ stained for $C 0$ showing the typical blob pattern. $\boldsymbol{E}$, Intrinsic signal map showing functional color domains. $\boldsymbol{F}$, Averaged $\mathrm{C} 0$ signal from 3 adjacent sections of layer $2 / 3$ (including section shown in $\boldsymbol{D}$ ) to increase signal-to-noise ratio. The red box marks a region of interest, shown in more detail in G-I. G, Color-coded CO blobs from 4 neighboring sections (each $100 \mu \mathrm{m}$ thick). Colors (yellow, red, blue, pink) indicate different sections. Contour lines of the averaged $(0$ blob signal are superimposed (white). $\boldsymbol{H}$ shows the depth-related displacement of cross sections of radial vessels in the $3 \mathrm{D}$ reconstruction. The displacement is a function of the angular orientation of the radial vessels and the cortical curvature. In $I$, the contours of the averaged $C 0$ blob signal are superimposed onto $\boldsymbol{H}$ to indicate the influence of this displacement on other radial structures such as the columnar $\mathrm{CO}$ blobs.

However, we found faint spots of increased stimulus responses in area V2 of cases M1 and M3 that roughly matched with patches seen in the red/green condition maps (see arrows). This colocalization is consistent with results reported for the macaque monkey that color and luminance stimuli activate the same functional regions in V2 (Roe et al., 2005a). 


\section{Alignment of functional data with anatomical data}

Functional anatomical studies are known to be affected by methodological difficulties in the alignment of the functional data to histologically processed preparations, as has been frequently stated by several authors (Polimeni et al., 2005; Sincich and Horton, 2005; Xiao et al., 2007; Adams and Horton, 2009). Different methodological approaches have been developed to overcome such difficulties. We used a multi modal vessel mapping approach described previously (Valverde Salzmann et al., 2011) to ensure accurate alignment of the functional and anatomical data (Fig. 3). An image of the superficial cortical vasculature labeled by a filling with fluorescent gelatin (Fig. 3A) was used as a precise template of the cortical physiology. Based on that template, distortions of sections were corrected by nonlinear image transformation algorithms. Thus, a three-dimensional reconstruction of the sectioned tissue was obtained (Fig. $3 B$ ) which allows for a highly precise alignment of functional maps (Fig. 3C,E) with histological data. The elaborate protocol of this approach allowed us to determine the tilt of radial structures within the imaged cortical region, e.g., of radial vessels descending from the surface of the cortex into deeper layers. Thereby we were able to determine and reduce the dislocation error of functional signals. In addition, this approach enabled us to determine the tilt of the columnar CO blobs (Fig. 3D) throughout the upper layers $2 / 3$. By averaging the CO signal intensities of the corresponding sections (Fig. $3 F$ ) we achieved a more precise localization of the CO blobs of layer $2 / 3$ (Fig. $3 G-I$ ) while taking the dislocation due to the tilt into account.

\section{Colocalization of functional color patches with $\mathrm{CO}$ blobs in visual area $\mathrm{V} 1$}

The functional color response maps of the red/green stimulus conditions from 2 animals (shown in Fig. $4 A, E$ ) were aligned to the corresponding histological CO signal maps (shown in Fig. $4 B, F)$. It is apparent that the locations of the functional color patches coincide with those of the CO blobs (Fig. $4 B, F$, see white arrows). The extent of the spatial overlap of functional color response with histological CO signals is presented in Figure 4, C and $G$, which plots the contours of the color response peaks superimposed onto the histological CO sections. The functional signals show a significant overlap with $\mathrm{CO}$ rich zones, as visualized in the color-coded overlays shown in Figure 4, D and $H$. We quantified this empirically evident colocalization of functional and histological blobs by calculating center-to-center distances between blobs within and across functional and histological maps. Functional color patches had a mean center-to-center spacing of $411.4 \pm 101.2 \mu \mathrm{m}(n=73)$ in case M1, of $405.7 \pm 85.1$ $\mu \mathrm{m}(n=103)$ in case M3 and of $448.8 \pm 80.1 \mu \mathrm{m}(n=95)$ in case M2.CO blobs had a mean center-to-center spacing of $417.3 \pm$ $88.3 \mu \mathrm{m}(n=62)$ in case M1 and of $416.5 \pm 87.8 \mu \mathrm{m}(n=60)$ in case M3. The dislocation of color patch and CO blob centers was $83.7 \pm 36.5 \mu \mathrm{m}(n=135)$ in case M1 and 85.5 $\pm 30.3 \mu \mathrm{m}(n=$ 162 ) in case M3. Consistent with previous studies on CO blobs and patch colocalization (Landisman and Ts'o, 2002; Lu and Roe, 2008), patches did not show a strict restriction to CO rich areas. However, we found that cases in which functional patches were fully disconnected from $\mathrm{CO}$ blob regions or vice versa were rare (see Fig. $4 D, H$ ).

Blob pattern formation across chromatic stimulus conditions 2DG studies show an activation of CO blob regions for a wide range of hue stimuli. Hue selective responses with patchy shapes in $\mathrm{V} 1$ have been reported in one OI study in macaques reported

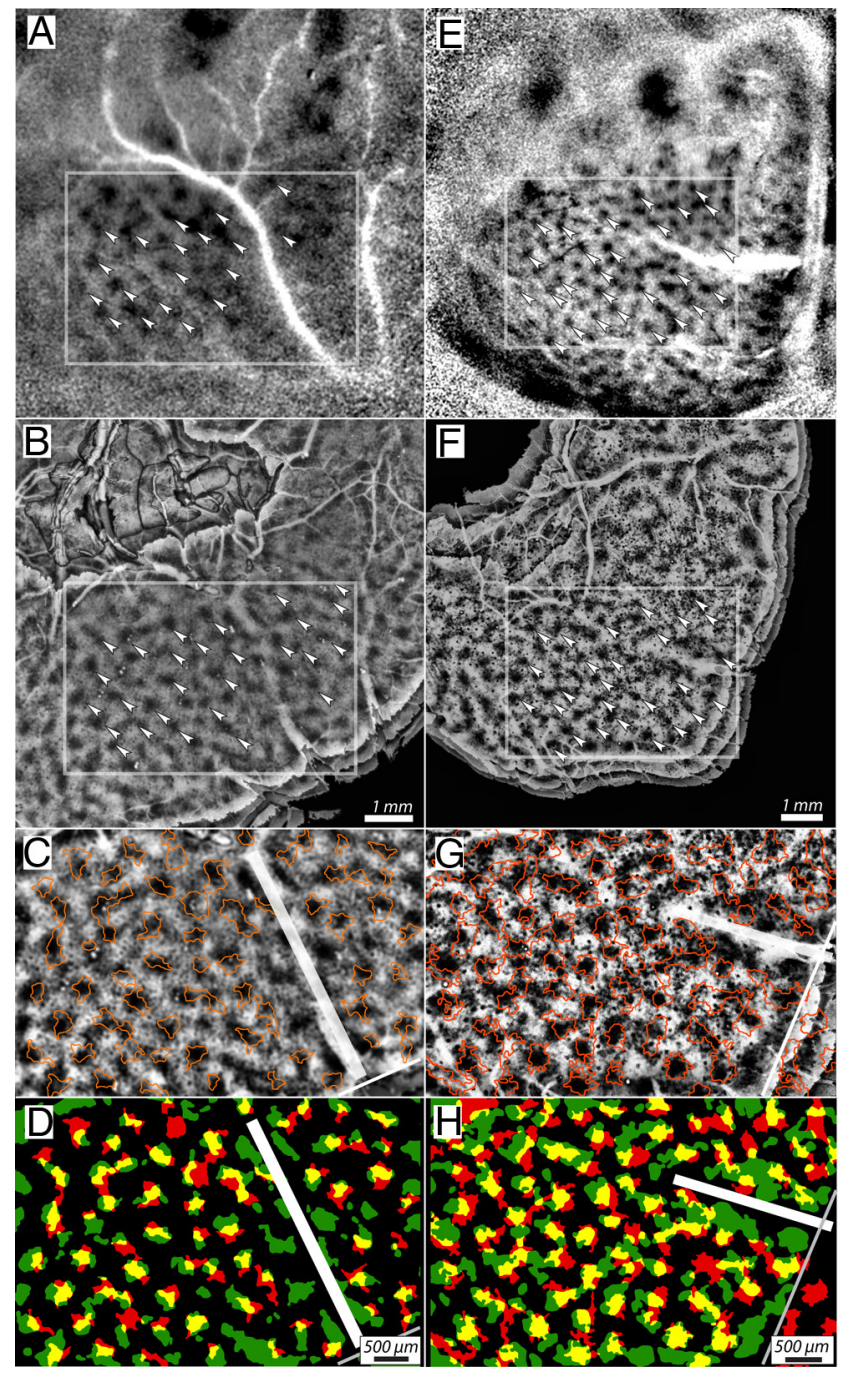

Figure 4. Alignment of functional color domains to CO blobs in 2 marmosets. $A-D$ show case $M 1, \boldsymbol{E}-\boldsymbol{H}$ show case $M 3 . \boldsymbol{A}, \boldsymbol{E}$, Intrinsic functional signal response to a red/green flicker, revealing color patches that are marked by arrows. $\boldsymbol{B}, \boldsymbol{F}$, Histological sections that were aligned to the functional maps of $\boldsymbol{A}$ and $\boldsymbol{E}$, respectively, showing $\boldsymbol{C} 0$ signal of the lower layer $2 / 3$. The arrows from $\boldsymbol{A}$ and $\boldsymbol{E}$ are superimposed on the maps in $\boldsymbol{B}$ and $\boldsymbol{F}$, revealing a colocalization of functional patches with CO blobs. $\mathbf{C} \boldsymbol{H}$, Magnification of the ROIs in $\boldsymbol{A}-\boldsymbol{F}$. $\boldsymbol{C}$ and $\mathbf{G}$ show the contour lines of the functional color domains (thin red lines) superimposed on the $C 0$ signal. Vessel positions are marked by the white thick lines. Section borders are indicated by thin white lines. $\boldsymbol{D}$ and $\boldsymbol{H}$ show the overlap (yellow) of functional domains (red) and C0-rich zones (green). The thick and thin white lines indicate vessel positions and section borders, respectively, as shown in $\mathbf{C}$ and $\mathbf{G}$.

by Xiao et al. (2007), but the histological proof for a colocalization with CO blobs was not given in that study. In contrast, color selectivity in V2 has been confirmed by several OI studies (Ts'o et al., 2001; Xiao et al., 2003; see also Tootell et al., 2004; Sincich and Horton, 2005). The 2DG studies suggest that a CO blob selective activation in V1 is predominantly achieved by stimuli consisting of red and blue hues, whereas stimuli consisting of green and yellow hues resulted in a faint labeling of CO blobs. Similar results could not be obtained by differential OI studies (Landisman and Ts'o, 2002) that found that reliable blob activation was achieved only by calculating the difference between red/green grating and achromatic grating stimulus responses.

Below we report the single-condition maps of all colorvarying conditions and the luminance-varying condition (Figs. 5, 6) to see whether a non-differential imaging approach (as indicated by the results of Xiao et al., 2007) might allow a more 
sensitive imaging of the cortical responses (Vanzetta et al., 2004; Polimeni et al., 2005).

Blob pattern formation in visual area $V 1$ Consistent with Landisman and Ts'o (2002) and $\mathrm{Lu}$ and Roe (2008) in macaques we found across all stimulus conditions the most obvious pattern formation with the red/green flicker conditions (shown in Fig. 5 for case M2, and in Fig. 6 for case M3). Flicker conditions in which red was set against isoluminant gray revealed an equally strong pattern formation. In stimulus conditions containing blue hues we found an activation pattern that was spatially overlapping with that obtained in red/green conditions but less prominent (Figs. 5, 6, blue/ yellow, blue/gray). In case M2 (Fig. 5) the pattern seen in the blue/yellow map shows a stronger pattern formation compared with the blue/gray flicker condition. The latter one shows only traces of the pattern and is almost unrecognizable to the untrained eye due to blood vessel noise. A more elaborate signal analysis might be able to outline such faint patterns in future studies. In contrast to case M2 we see no patchy pattern in the blue/yellow conditions of case M3 (Fig. 6), but the map shows a similar pronunciation of large superficial blood vessels that dominate the response map as seen in the blue/ gray condition of M2. However, the blue/ gray flicker stimulus shows an equally faint blob pattern formation in M3 as seen in the blue/gray condition of case M2.

Green/gray and yellow/gray flicker conditions did not reveal any obvious pattern. Neither did black/white flicker conditions, nor single-condition maps of orientation selective responses (Figs. 5, 6; $90^{\circ}$ ) when calculated in the same way as the color condition maps. With respect to the stimulus specificity for cone activation and the presented pattern formation tendencies we were able to demonstrate that $\mathrm{CO}$ blob activation as seen in the singlecondition maps demonstrated here was mainly triggered by color-varying stimuli rather than by changes in luminance.

Relative contributions of $(L-M), S$, and $(L+M)$-cone contrasts to blob pattern formation in $V 1$

Following the above observations that indicate hue-dependent, but not luminance-dependent patch formation, we were interested to determine the degree to which the formation of color patches could be accounted for by activation of the different L,M, $\mathrm{S}$ cone types. We calculated the influence of each hue of the chromatic stimuli on the L-, M-, and S-cones by taking the dot product of the stimulus spectra and the estimated cone spectral sensitivity specific to marmoset cones (Forte et al., 2006). Table 2 shows the resulting raw L,M,S-cone activation values, for easier readability normalized to a maximum of 1 obtained with the

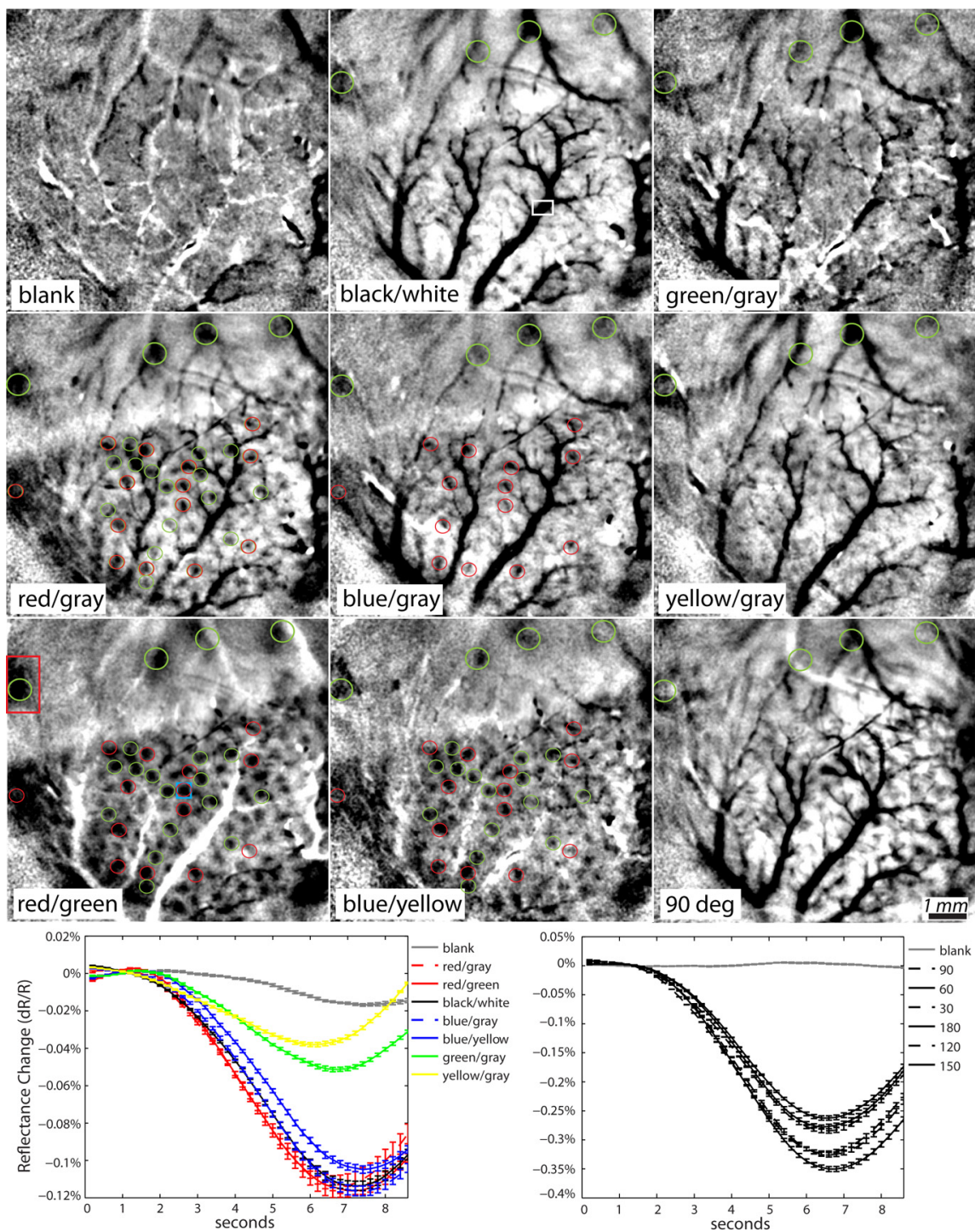

Figure 5. Comparison of intrinsic signal responses of case $M 2$ to color-varying and luminance-varying stimuli. The blank condition shows the control for the non-stimulus condition (gray screen). Red/green, red/gray flicker conditions show clear patchy patterns. A region of interest is marked in red to show subdomains in V2 responses (see Fig. 11). The blue/yellow flicker condition reveals the same pattern but is less prominent. Maps from the blue/gray conditions show only faint pattern formation. Small red and green circles mark exemplary positions of activated color domains. A single-condition map of the intrinsic signal response to a vertical grating $\left(90^{\circ}\right)$ stimulus is presented for comparison. No pattern of orientation domains as seen in differential maps is expressed. The $90^{\circ}$ map shows a similar appearance as the black/white flicker condition map. In the latter one, a white rectangle indicates the region of interest for calculating vessel reflectance changes throughout conditions. Large green circles mark the position of color domains in V2 throughout all stimulus conditions. In green/gray, yellow/gray and black/white conditions such rectangle in red/green condition) in response to color-varying stimuli, and at the lower right plot the intrinsic signal time courses from the same region of interest in response to achromatic grating stimuli ( 6 orientations) are presented. The time courses are normalized to frame zero. For more details, see Table 3 and Blob pattern formation and signal amplitude.

white stimulus. Neural activity observed in our seven stimulus conditions would be expected to be related to absolute modulations of L,M,S-cone contrasts or to differences thereof, referred to as cone contrast axes, defined as $(\mathrm{L}-\mathrm{M})$ (red/green), S (blue), $\mathrm{S}-(\mathrm{L}+\mathrm{M})$ (blue/yellow), (L+M) (luminance), elicited by the alternation of the two hues contained in each flicker stimulus. Cone contrasts were calculated as cone activation for one stimulus relative to a background stimulus, resulting in fractional changes [i.e., cone contrast $=$ (cone activity stimulus - cone activity background)/cone activity background]. Since we alternated between two hues (or between white and black), we used 


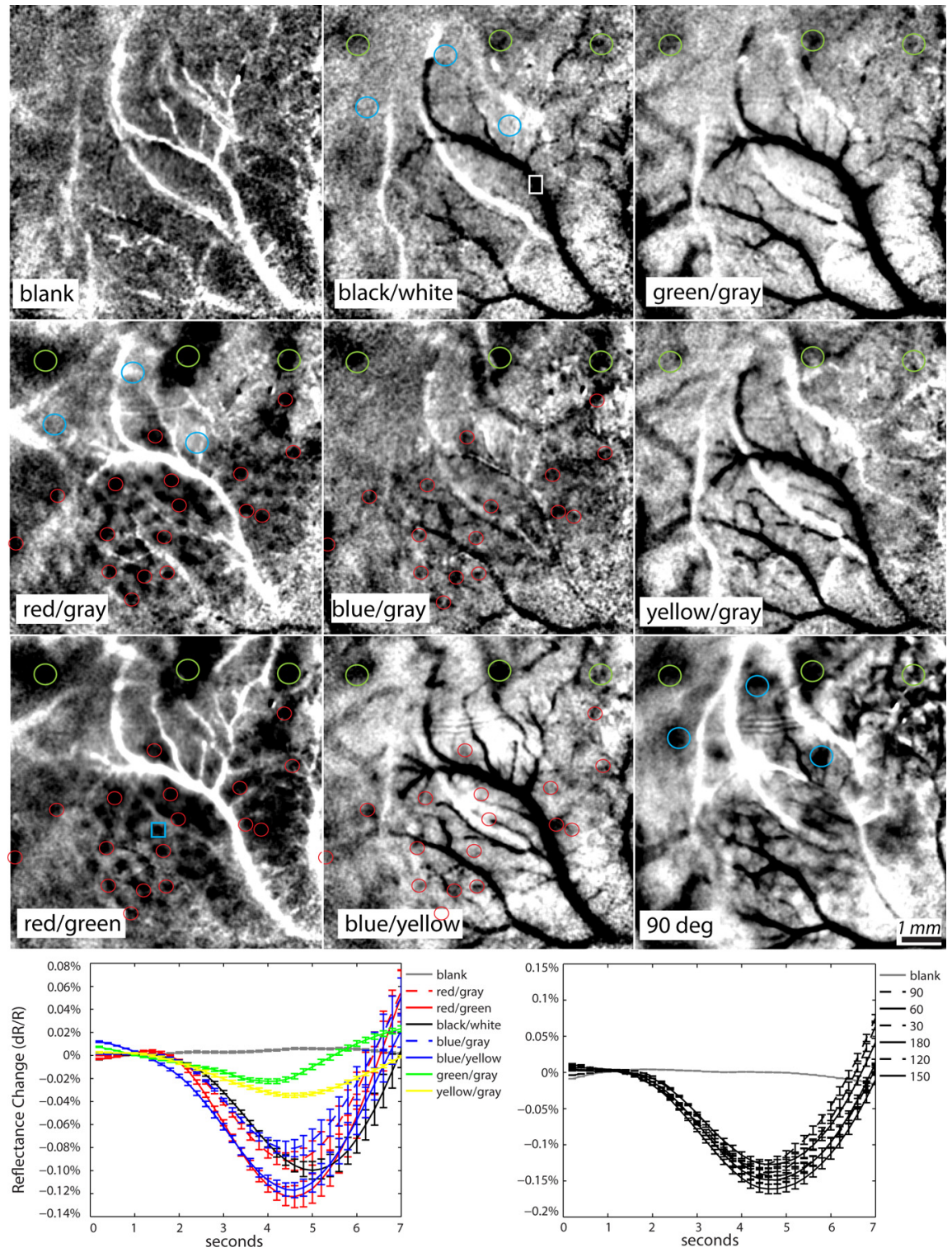

Figure 6. Comparison of intrinsic signal responses of case M3 to color-varying and luminance-varying stimuli. The blank condition shows the control for the non-stimulus condition (gray screen). Red/green, red/gray flicker conditions show clear patchy patterns. Maps from the blue/gray conditions show a faint expression of color domains. The blue/yellow flicker condition revealed no obvious pattern. Small red circles mark exemplary positions of color domains, blue circles in the red/gray condition map mark locations of orientation response patches $\left(\right.$ see $90^{\circ}$ ). A single-condition map of the intrinsic signal response to a vertical grating $\left(90^{\circ}\right)$ stimulus is presented. No pattern of orientation domains as seen in differential maps is expressed. Large green circles mark the position of color domains in V2 throughout all stimulus conditions. In green/gray, yellow/gray and black/white conditions such regions show less activation. Blue stars in the $90^{\circ}$ condition map shows orientation selective domains in V2 not expressed in chromatic conditions nor in the black/white flicker conditions. In the latter one, a white rectangle indicates the region of interest for calculating vessel reflectance changes throughout conditions. Lower left plot shows the intrinsic signal time courses of a color domain in V1 (see blue rectangle in red/green condition) in response to color-varying stimuli, and at the lower right plot, the intrinsic signal time courses from the same region of interest in response to achromatic grating stimuli (6 orientations) are presented. The time courses are normalized to frame zero. For more details, see Table 3 and Blob pattern formation and signal amplitude.

the mean cone activation between the two flickered hues as background for the calculation. Figure 7A shows cone contrasts for a selection of stimulus pairs used here. Activity along cone contrast axes was calculated as the difference between the relevant cone contrasts. Figure $7 B$ shows the absolutes of cone contrast axes modulations for each stimulus pair.

The red/green flicker stimulus led as expected to maximal (and selective) modulation of the $(\mathrm{L}-\mathrm{M})$-cone contrast axis, which coincided with the pronounced patch formation tendency seen under the red/green stimulus condition. However, while our red/green stimulus was highly selective for the $(\mathrm{L}-\mathrm{M})$-cone modulation (with near absence of S-cone modulation), we have to acknowledge that the blue/yellow stimulus did not yield the same high specificity for S-cone stimulation, as it also involved modulation along the $(\mathrm{L}-\mathrm{M})$-axis. Thus the fainter patch formation seen in blue/yellow and blue/ gray conditions may also be due to a modulation of the $(\mathrm{L}-\mathrm{M})$-axis. However, the green/gray stimulus pair, for which patchformation was absent, also had nearabsent ( $\mathrm{L}-\mathrm{M})$-axis modulation, and instead drove almost exclusively the $S$ and $\mathrm{S}-(\mathrm{L}+\mathrm{M})$ axes. The other stimulus pairs modulated the different color axes to varying degrees. Thus, the multitude of seven flicker stimuli, each with a different involvement of the color-axes $(\mathrm{L}-\mathrm{M}, \mathrm{S}$, $\mathrm{L}+\mathrm{M}$ ), allowed us to quantify to which extent patch formation was driven by each of the color-axes in context of a general linear model (GLM). For this purpose we measured the difference in IOI signal between patch and interpatch for each of the seven stimuli across both monkeys, and regressed the patch-interpatch difference against the L,M,S-cone contrast modulations evoked by the seven flicker stimuli. The patch-interpatch differences for the seven stimuli were $Z$-normalized for each animal and then concatenated. The GLM thus modeled 14 data points by estimating $\beta$-weights for three regressors. Patch and interpatch activity was extracted as follows. In both animals we defined a mask for blob and interblob areas based on locations of patch and interpatch areas in the blue/yellow condition of case 2 and blue/gray condition of case 3 . Note that we avoided red/green-flicker-evoked activity for the definition of blob and interblob masks to avoid data-selection. The masks were defined using the central region of the color domain maps shown in Figures 5 and 6 and were used to extract activity related to all flicker stimulus conditions. The blob-mask consisted of disks of $150 \mu \mathrm{m}$ diameter centered on 8 (in case 2) and 11 (in case 3 ) color patches that were identified by eye and that showed no obstructions e.g., by blood vessel noise. Interblob masks consisted of ring shaped areas with a diameter of $650 \mu \mathrm{m}$ and a ring width of $200 \mu \mathrm{m}$ that encircled the corresponding patch without overlapping any neighboring patch. Neighboring patches that overlapped with interblob areas were masked with a $250 \mu \mathrm{m}$ disk to exclude them. Values for blobs and interblobs were averaged separately for each stimulus condition and for each animal. Thus, the subsequent GLM analysis allowed us to estimate the degree to 
Table 2. Cone activation: estimated cone activation by the color-varying stimuli and luminance-varying stimuli used

\begin{tabular}{|c|c|c|c|c|c|c|c|c|}
\hline \multicolumn{7}{|c|}{ Color varying } & \multicolumn{2}{|c|}{ Luminance varying } \\
\hline Cone type & Blue & Yellow & Blue & Gray & Yellow & Gray & Black & White \\
\hline$\llcorner 563$ & 0.114 & 0.0662 & 0.1147 & 0.0550 & 0.8689 & 0.9294 & 0.0012 & 1.0000 \\
\hline L556 & 0.1373 & 0.0646 & 0.1381 & 0.0551 & 0.8456 & 0.9280 & 0.0012 & 1.0000 \\
\hline M543 & 0.1909 & 0.0610 & 0.1920 & 0.0554 & 0.7917 & 0.9265 & 0.0013 & 1.0000 \\
\hline 5423 & 0.9327 & 0.0073 & 0.9366 & 0.0656 & 0.0467 & 0.9418 & 0.0023 & 1.0000 \\
\hline
\end{tabular}

Color varying

\begin{tabular}{|c|c|c|c|c|c|c|}
\hline Cone type & Red & Green & Red & Gray & Green & Gray \\
\hline$\angle 563$ & 0.2233 & 0.1930 & 0.2250 & 0.2116 & 0.1866 & 0.2014 \\
\hline L556 & 0.1822 & 0.1993 & 0.1836 & 0.2119 & 0.1927 & 0.2016 \\
\hline M543 & 0.1146 & 0.2057 & 0.1155 & 0.2130 & 0.1989 & 0.2027 \\
\hline S423 & 0.0161 & 0.0144 & 0.0146 & 0.2474 & 0.0135 & 0.2338 \\
\hline
\end{tabular}

The cone type $L 563$ was not present in our animals and is plotted for completeness in italics.

A L,M,S cone contrasts for color opponent stimuli

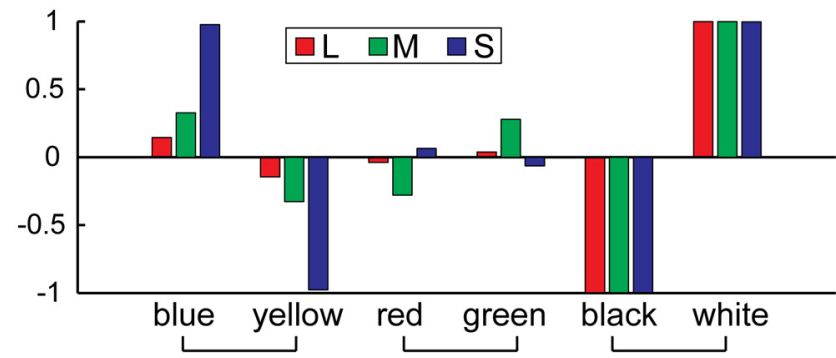

B Color axis modulations by each stimulus pair

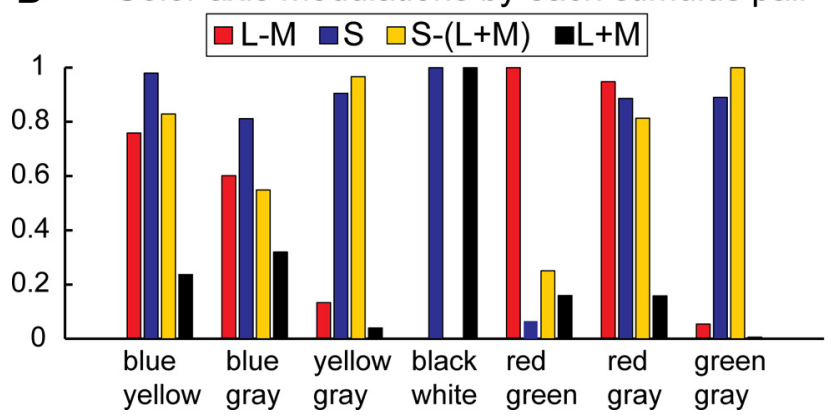

Figure 7. Estimated stimulus-induced L,M,S-cone contrast modulation. A, L,M,S-cone contrasts evoked by the different hues used in this study, shown for a selection of stimulus pairs. Cone contrast was calculated relative to the mean cone activity evoked by both stimuli of a given stimulus pair, hence the symmetry for each pair (pairs are indicated by the underlying bracket). Note that results for subsequent analyses did not differ when contrasts were calculated using gray background. $\boldsymbol{B}$, Modulation of cone contrast axes for each stimulus pair. For each stimulus pair the absolute of the difference of cone contrast axis modulation is shown along four cone contrast axes. For easier comparison, each cone contrast axis is normalized to a maximum of 1.

which each (L,M,S)-cone contrast modulation contributed (additively and linearly) to the patch-interpatch difference obtained in the IOI signal across the seven flicker stimuli. The GLM provided a surprisingly good fit, in that its three regressors accounted for $80 \%$ of the variance of the patch-interpatch activity difference ( $F$ test: $p<0.0009$; Fig. $8 A$ ). The same results show that most of the variance is explained by $\mathrm{L}$ - and $\mathrm{M}$-cone contributions with opposite signs, with comparably little contribution of the S-cone contrast (Fig. $8 B$ ). We thus ran a mathematically equivalent regression model, using the modulation along the cone contrast axes $(\mathrm{L}-\mathrm{M}, \mathrm{S}, \mathrm{L}+\mathrm{M})$ as regressors. This model is equivalent as it uses linear combinations of the regressors of the first model, thus also explaining $80 \%$ of the variance. This regression showed that the $(\mathrm{L}-\mathrm{M})$-axis was the only regressor accounting for a significant amount of variance, as only the removal of the $(\mathrm{L}-\mathrm{M})$ -

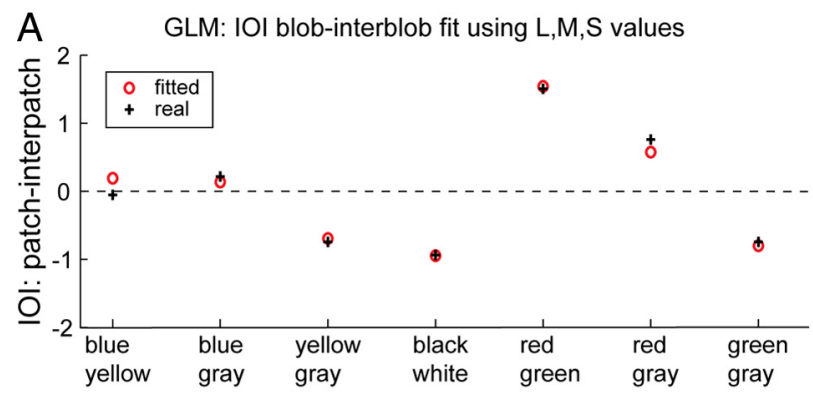

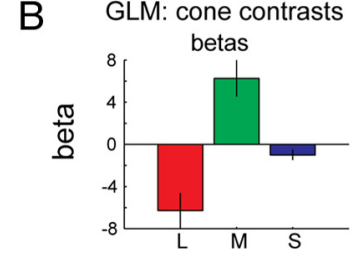

D $\mathrm{R}^{2}$ loss by removal of predictors

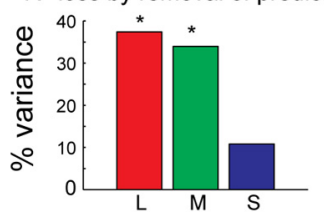

C

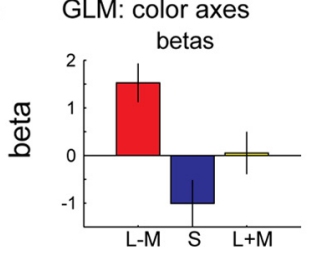

E $\mathrm{R}^{2}$ loss by removal of predictors

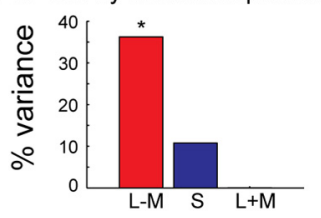

Figure 8. GLM fit between observed patch formation in V1 and calculated L,M,S-cone contrast modulations evoked by the stimulus pairs. $A, 10$ I patch-interpatch differences Z-normalized and averaged across monkeys, plotted for each color-stimulus pair (black crosses). Red circles show the fit using a GLM containing as three regressors the L,M,S-cone contrast modulations elicited by each stimulus pair. The fit explains $80 \%$ of the 10 l variance $(p<0.0009)$. B, $\boldsymbol{C}, \beta$-estimates for a GLM model using L,M,S-cone contrasts as regressors $(\boldsymbol{B})$ and $\beta$-estimates of a GLM model using $(L-M), S,(L+M)$ (i.e., cone contrast differences) as regressors (C). D, E, Percentage loss of variance explained by the model (i.e., loss of $R^{2}$ ) when single regressors are removed from the full models. Only $L, M$ or $(L-M)$ account for significant variance. ${ }^{*} p<0.01,{ }^{* *} p<0.0001$ (F tests).

predictor from the full model lead to a significant loss in the variance explained ( $F$ test: $p<0.0037$, Fig. $8 E$ ), with no significant loss of variance when the S-predictor was removed. Equivalently, only removal of either L- or M-predictors in the first model led to significant loss of variance, but not removal of the S-predictor (Fig. 8). [Note that equivalent results were obtained when the S-predictor was replaced by a $S-(L+M)$ predictor; data not shown here.] In sum, this quantitative analysis of L,M,S-cone contrast contributions to patch formation shows that it was the $(\mathrm{L}-\mathrm{M})$-cone contrast modulation that drove the patch formation across the seven flicker stimuli (including the blue/yellow 
Table 3. Response amplitudes for all stimulus conditions: intrinsic signal response amplitudes for the different stimulus conditions

\begin{tabular}{|c|c|c|c|c|c|c|c|c|}
\hline & Red/green & Blue/yellow & Red/gray & Green/gray & Blue/gray & Yellow/gray & Black/white & Mean grating \\
\hline \multicolumn{9}{|c|}{ Peak response amplitude $(\Delta R / R)$} \\
\hline Case M2 & $-0.116 \%$ & $-0.104 \%$ & $-0.114 \%$ & $-0.051 \%$ & $-0.116 \%$ & $-0.038 \%$ & $-0.113 \%$ & $-0.303 \pm 0.033 \%$ \\
\hline Case M3 & $-0.061 \%$ & $-0.058 \%$ & $-0.045 \%$ & $-0.011 \%$ & $-0.040 \%$ & $-0.017 \%$ & $-0.049 \%$ & $-0.140 \pm 0.012 \%$ \\
\hline Case M1 & $-0.178 \%$ & & & & & & $-0.115 \%$ & $-0.291 \pm 0.02 \%$ \\
\hline \multicolumn{9}{|c|}{$(\Delta R / R)$ normalized to mean grating amplitude } \\
\hline Case $\mathrm{M} 2$ & 0.383 & 0.343 & 0.376 & 0.168 & 0.383 & 0.125 & 0.373 & 1 \\
\hline Case M3 & 0.436 & 0.414 & 0.321 & 0.079 & 0.286 & 0.121 & 0.350 & 1 \\
\hline Case M1 & 0.612 & & & & & & 0.395 & 1 \\
\hline
\end{tabular}

flicker, that contained considerable ( $\mathrm{L}-\mathrm{M})$-modulation), with only little influence of the $\mathrm{S}$ and no influence of the $(\mathrm{L}+\mathrm{M})$ (luminance) axis.

Blob pattern formation in visual area $V 2$

As in V1, we found the strongest signal responses in V2 for red/ green and blue/yellow flicker stimuli. In red/green and red/gray conditions large elongated patches were revealed. The blue/yellow and blue/gray conditions showed congruent patches of smaller size. Green/gray and yellow/gray conditions also showed congruent activation patches. However these patches resembled those V2 activation patches that were revealed in the black/white flicker conditions. Note that for technical reasons in case M2 (Fig. 5) cortical area V2 was slightly out of focus. Thus, the signals from this area were less intense and the noise of the blood vessels was somewhat more dominant. Nevertheless, the relative signal intensities evoked by the different color stimuli were consistent with the pattern formation in V1, and also consistent with those observed in case M3.

\section{Blob pattern formation and signal amplitude}

The intrinsic signal time course of the chromatic stimulus conditions in case M2 (Fig. 5, left plot) and case M3 (Fig. 6, left plot) roughly reflects the pattern formation relations of the singlecondition maps. Stimulus conditions containing red or blue hues showed similar strength in response amplitudes (Table 3). Pattern formation seems to correlate with the signal amplitude in these cases (except for the blue/gray condition in case M3 which shows a more obvious pattern but smaller signal amplitude than the corresponding blue/yellow condition). Peak response amplitudes of green/gray and yellow/gray conditions were less than half the size of the amplitudes of stimulus conditions containing red or blue hues. Summing the red/gray, green/gray, blue/gray and yellow/gray responses we end up with a value (case M2 $=0.319 \%$, case M3 $=0.113 \%$ ) close to the one measured for the mean amplitude of the orientation selectivity response (Table 3 ). In all cases, the largest chromatic response amplitude observed is in between 38 and $61 \%$ of the size of the corresponding grating response amplitude.

Vascular noise and signal

Figures 5 and 6 show-apart from functional patch formationalso large superficial blood vessels, mostly veins. Berwick et al. (2005) show that large $\mathrm{HbO}_{2}$ responses can be found at the large surface draining veins. We therefore applied our GLM analysis to vein-ROIs (Figs. 5, 6) to examine the strength of stimulus-related functional signal components that may derive from accumulating capillary blood. The results were qualitatively similar to those observed for the patch-interpatch difference, yet weaker. The full GLM accounted for $62 \%$ of total signal variance ( $F$ test: $p=0.02$ ), and the regressors coding for $\mathrm{L}$ - and $\mathrm{M}$-cone contrasts (as well as the $\mathrm{L}-\mathrm{M}$ regressor in the cone contrast axis model) achieved significances of $p<0.05$ each. The results suggest that draining veins can carry functional signals related to patches, which in turn may be related to fMRI voxel-pattern formation (Seymour et al., 2009; Shmuel et al., 2010). On the other hand, these results are to be considered very preliminary, as more detailed analyses (e.g., of time courses), as well as new experiments such as $2 \mathrm{D}$ spectroscopy at high frame-rates would be required for a comprehensive understanding of the reasons underlying the observed effect, including the exclusion of potential vasomotion artifacts (Mayhew et al., 1996; Berwick et al., 2005). While this is beyond the scope of the present study, this may be worthwhile, in particular in view of the implications for the interpretation of fMRI data.

\section{Ocular dominance and color patches}

Ocular dominance and CO blobs are highly correlated in macaque monkeys. CO blobs tend to line up with centers of ocular dominance columns (ODCs) and each CO blob is dominated by one eye or the other (for review, see Adams and Horton, 2009). Landisman and Ts'o (2002) and Lu and Roe (2008) confirmed this relation of ocular dominance and color patches in macaque monkeys using OI. In contrast New World monkeys neither show a strict relationship between CO blobs and ODCs, nor do they necessarily reveal ODCs (Spatz, 1989; Adams and Horton, 2009). Roe et al. (2005b) showed that weak ODCs can be imaged in some marmosets. We looked for differences in color domain maps obtained in two animals by binocular and monocular stimulus presentation. In both cases (M1 and M2) pattern formation due to red/green stimuli could be observed in the monocular conditions, but no differences in the location or spacing of the functional domains obtained using binocular or monocular stimulation were found. In Figure 9, $A$ and $B$, the marked domains (small red and green stars) of the monocular signal map showed the same nearest neighbor domains as the corresponding domain of the binocular signal map. Thus, it is very unlikely that only a subset of domains was selectively activated in these animals, in contrast to what was shown by Lu and Roe (2008; see their Fig. 7) for the macaque monkey where the monocularly activated blobs form rows within the ODCs of the stimulated eye.

In case M1, the signal amplitude of the chromatic stimulus conditions showed an equally strong signal amplitude for bothmonocular and binocular-chromatic and achromatic flicker conditions (Table 4). Only the peak amplitudes of the achromatic grating conditions differed significantly. In case $\mathrm{M} 2$, the peak amplitudes of red/green, blue/yellow, blue/gray, red/gray, black/ white flicker conditions differed by $<0.023 \%$. In the red/green, blue/yellow, black/white and blue/gray conditions the signal amplitude of the monocular conditions even exceeded the one from the corresponding binocular conditions. Significant differences in signal amplitude were apparent in the green/gray and yellow/ 

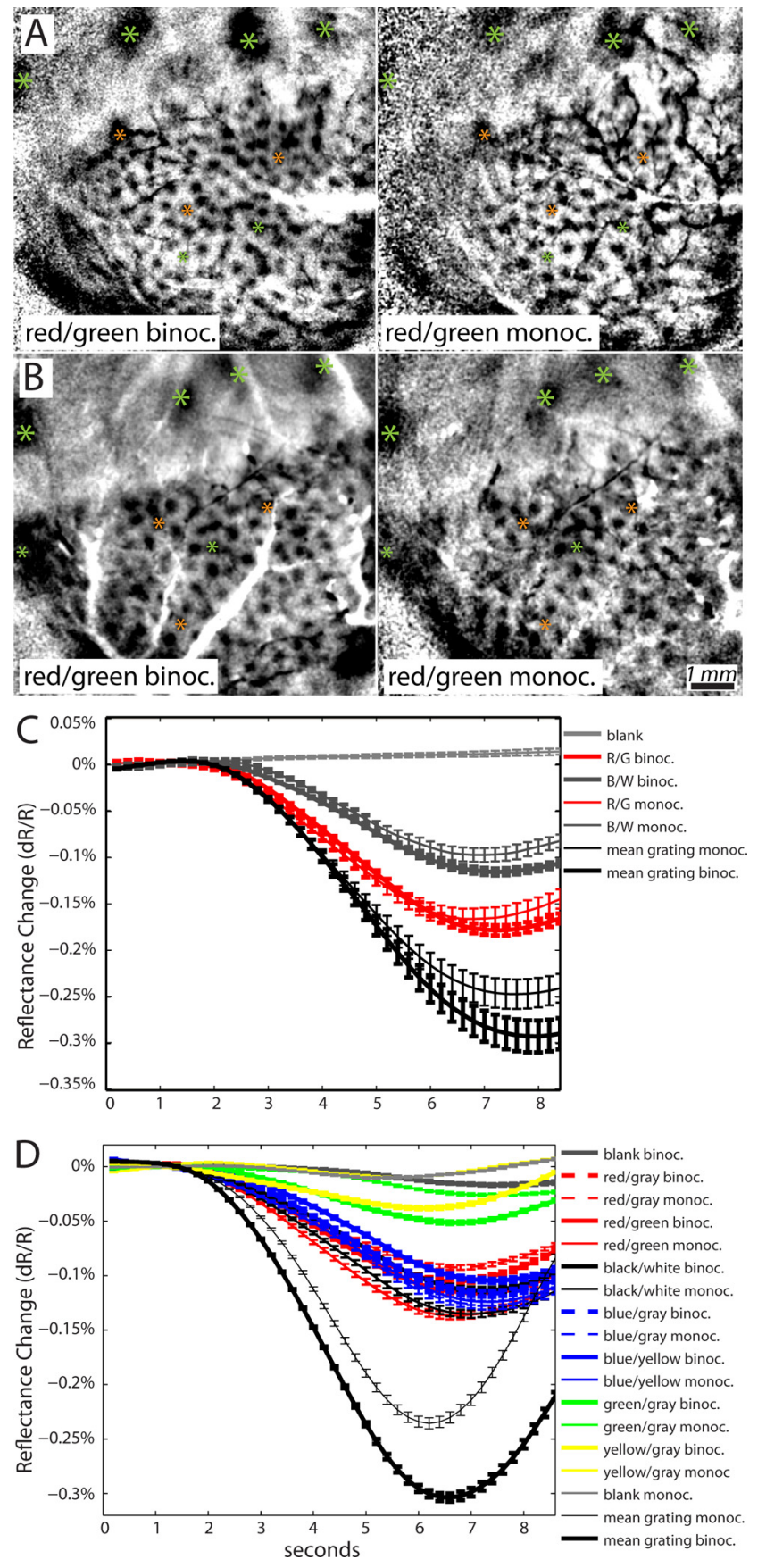

Figure 9. Color domains in single-condition maps of monocular and binocular stimulation. $\boldsymbol{A}, \boldsymbol{B}$, The pattern in the monocular condition maps ( $\boldsymbol{A}$, case M1; $\boldsymbol{B}$, case M2) shows the same response peak distribution as that seen in the binocular condition, thus indicating that blobs in marmosets are not subject to ocular dominance. $\boldsymbol{C}, \boldsymbol{D}$, Binocular versus monocular color responses. ( shows time courses of the intrinsic signal response in case M1 to binocularly and monocularly viewed achromatic gratings (black), red/green flicker (red), and black/white flicker (dark gray) (data normalized to frame zero). D, Time courses of the intrinsic signal response in case M2 to binocular and monocular stimulation with the full set of chromatic stimuli and achromatic gratings. For details see also Table 4.

gray conditions and the achromatic grating stimulus conditions (see mean grating response in Fig. 9C,D; Table 4).

\section{Color responses in V2}

$\mathrm{V} 1$ and V2 are clearly demarcated in the intrinsic signal maps of the chromatic stimulus conditions (Fig. 10A,F) by the abrupt change in spatial frequency of the functional pattern. The corre- sponding $\mathrm{CO}$ stain (Fig. 10C,H) shows an even more abrupt border between V1 and V2. The small color patches of V1 tend to cross the $\mathrm{CO}$ border at regular intervals to extend to some amount into V2 (see arrows Fig. $10 A, F$ ). These extensions line up with large signal patches that are spaced at intervals of 2-2.5 $\mathrm{mm}, 1-2 \mathrm{~mm}$ away from the border V1/V2. The alignment with the CO staining pattern (Fig. $10 E, J$ ) shows a colocalization of the patches with the thin stripes that run roughly orthogonal to the border V1/V2 (Horton, 1984). However there is no obvious relation between CO dark regions and color patches. The contours of the patches indicate a further segmentation of the large patches into smaller functional subdomains (Fig. 10G).

\section{Spatial organization of hues in $\mathrm{V} 2$}

Unlike color representation in V2 of the macaque monkey, the spatial organization of the color patch subdomains of V2 in the marmoset did not reveal the regular representation of the DIN (Deutsches Institut für Normierung e.V.) color system (Xiao et al., 2003). Instead we find that centers of different color patch subdomains and the patches of black/white conditions clustered within small cortical regions (Fig. 11). We observed that patches evoked by color-varying stimuli consisting of color opponent hue pairs revealed a stronger segregation into subdomains compared with those evoked by color-varying stimuli consisting of a single hue set against gray [see red/gray versus red/green, blue/gray versus blue/yellow, Fig. $11(\mathrm{y})$ ]. This suggests that color opponent stimulation was advantageous to increase pattern formation in functional maps. However, contours of all color-varying stimulus responses appeared to be congruent. This could indicate that the same subdomains were activated, but we cannot rule out that the lack of spatial organization of hues in our maps is due to differences in signal strength among chromatic conditions.

\section{Discussion}

\section{Summary}

In the visual cortex of three female trichromatic marmosets, we found domains of cortical response to isoluminant color stimuli. Red/green flicker of low temporal frequency $(1.5 \mathrm{~Hz})$ resulted in the strongest signal pattern formation. Blue/yellow flicker led to less prominent patterns. Blue/gray and red/gray flicker showed equal pattern formation as blue/yellow and red/green flicker. Patch formation was predominantly driven by the $(\mathrm{L}-\mathrm{M})$-cone contrast color axis of the stimuli, with a marginal contribution of the S-cone contrast axis. Importantly, we found color domains in $\mathrm{V} 1$ to be congruent with areas of increased $\mathrm{CO}$ reactivity. The functional patterns are consistent in monocular and binocular conditions, suggesting that blobs in marmosets are not subject to ocular dominance. In V2, color domains showed colocalization with thin stripes. Thus, our results are highly consistent with previous studies that show color domains in the visual cortex of macaques (Tootell et al., 1988, 2004; Tootell and Hamilton, 1989; Landisman and Ts'o, 2002; Lu and Roe, 2008).

\section{CO pattern and color patches}

We found similar center-to-center distances among CO blobs (416-417 $\mu \mathrm{m})$ and among color patches (404-448 $\mu \mathrm{m})$, and a tight colocalization between CO blobs and color patches, demonstrating a significant colocalization of the functional and anatomical columns. However, we found a small dislocation error between CO blob and color patch centers $(\sim 83-85 \mu \mathrm{m})$, which is around half the size of the error $(200 \mu \mathrm{m})$ found by Landisman and Ts'o (2002) in macaques. We believe that the smaller error is mainly due to our elaborate alignment approach. We also find 
Table 4. Response amplitudes for monocular versus binocular stimulus conditions: intrinsic signal response amplitudes for monocular versus binocular stimulus conditions

\begin{tabular}{|c|c|c|c|c|c|c|c|c|}
\hline & Red/green & Blue/yellow & Red/gray & Green/gray & Blue/gray & Yellow/gray & Black/white & Mean grating \\
\hline \multicolumn{9}{|l|}{ Case M2 } \\
\hline Monocular & $-0.136 \%$ & $-0.124 \%$ & $-0.092 \%$ & $-0.026 \%$ & $-0.127 \%$ & $-0.010 \%$ & $-0.135 \%$ & $-0.235 \pm 0.0045 \%$ \\
\hline Binocular & $-0.116 \%$ & $-0.105 \%$ & $-0.105 \%$ & $-0.051 \%$ & $-0.116 \%$ & $-0.038 \%$ & $-0.113 \%$ & $-0.303 \pm 0.0033 \%$ \\
\hline \multicolumn{9}{|l|}{ Case M1 } \\
\hline Monocular & $-0.166 \%$ & & & & & & $-0.097 \%$ & $-0.247 \pm 0.015 \%$ \\
\hline Binocular & $-0.178 \%$ & & & & & & $-0.116 \%$ & $-0.292 \pm 0.017 \%$ \\
\hline
\end{tabular}

Peak response amplitude $(\Delta R / R)$.

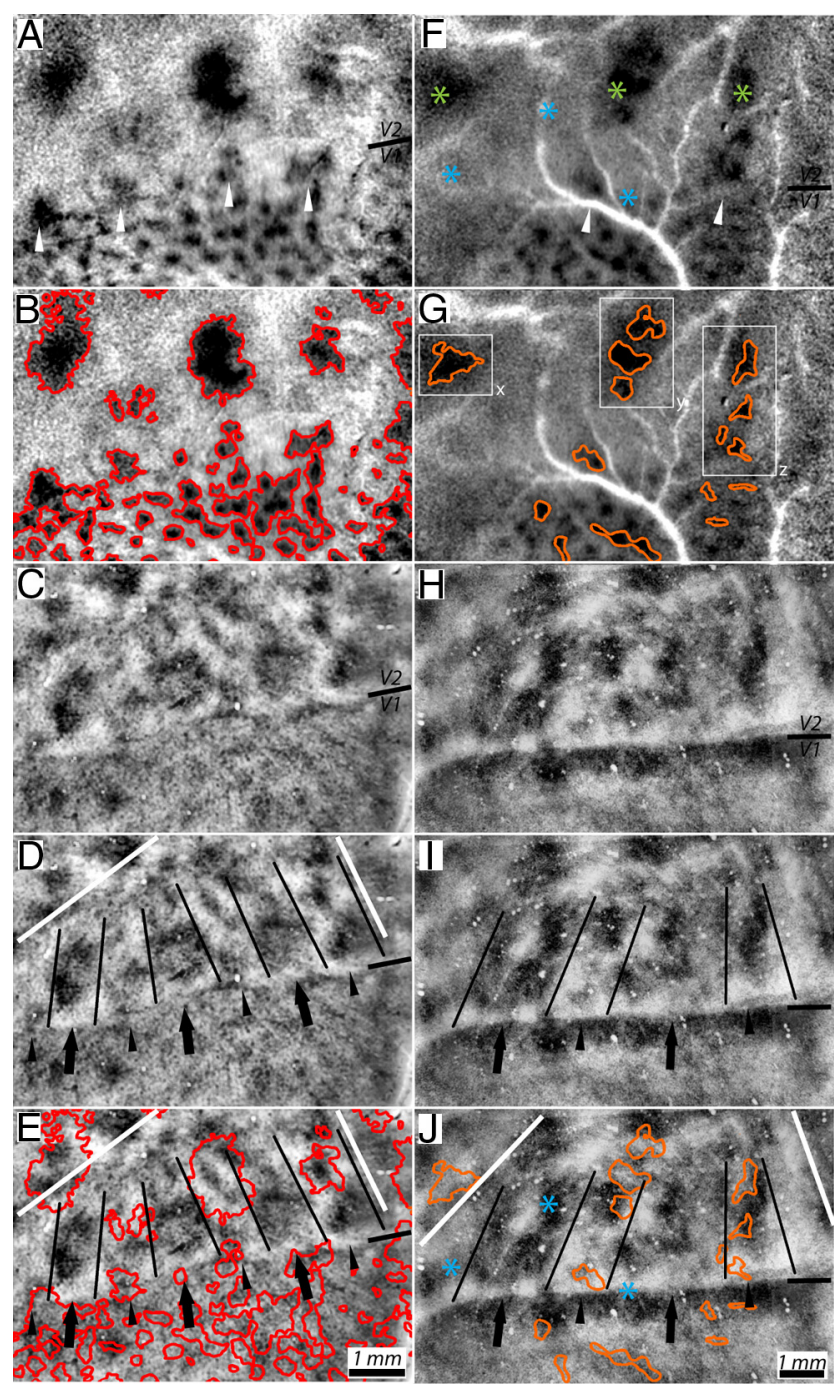

Figure 10. Color response in V2.A-J, Alignment of red/green color patches and CO reactivity pattern of case M1 $(\boldsymbol{A}-\boldsymbol{E})$ and of case $\mathrm{M} 3(\boldsymbol{F}-\boldsymbol{J}) . \boldsymbol{A}$ and $\boldsymbol{F}$ show the red/green flicker response map. White arrows mark regions where the domain pattern of $\mathrm{V} 1$ extends into V2. Blue stars mark positions of patches from the achromatic grating condition of Figure $6\left(90^{\circ}\right) . B, G$, Contours of the color response. Three regions of interest $(x, y, z)$ are marked in $\boldsymbol{G}$ which correspond to contour maps in Figure 11. $\boldsymbol{C}-\boldsymbol{E}$ and $\boldsymbol{H}-\boldsymbol{J}$ show the $\mathrm{C}$ reactivity pattern of $\mathrm{V} 2$. In $\boldsymbol{D}$ and $\boldsymbol{I}$, thick and thin stripes are indicated by thick and thin arrows respectively. $E, J$, Contours of color patches from $\boldsymbol{B}$ and $\boldsymbol{G}$ superimposed onto V2. Patch contours indicated a colocalization of color responses and thin stripes. Blue stars show that patches exclusively found in achromatic grating conditions do not fall into thin stripe regions.

smaller distances of CO blobs as reported for macaques (350-550 $\mu \mathrm{m})$ and humans (0.75-1 mm) (Horton and Hubel, 1981; Horton and Hedley-Whyte, 1984). With respect to the smaller brain size of marmosets and variability of the functional anatomy of the visual areas in primates (Adams and Horton, 2009) these differ- ences appear consistent and are further corroborated by the similar size of the center-to-center distance of color patches and the blob density in marmosets of $3.9-5.5 \mathrm{blobs} / \mathrm{mm}^{2}$ reported by Solomon (2002). Likewise, consistent with previous studies (Tootell and Hamilton, 1989; Tootell et al., 2004; Lu and Roe, 2008), color patches in V2 of marmosets do not show any obvious correlation to regions of high CO density but appear as irregular bands or elongated patches that are oriented perpendicular to the border V1/V2. We find that these color patches are made up of smaller subdomains that vary in size and distribution among the different chromatic conditions. Unlike Xiao et al. (2007), we do not find a spatially organized representation of hues. Subdomains of different chromatic conditions show largely overlapping shapes suggesting a common structural origin. However, the sizes of the individual subdomains correlate with the strength of the intrinsic response. Thus, it is possible that the lack of spatial organization seen in our maps is due to differences in signal strength among chromatic conditions. The use of imaging protocols that are able to equalize the stimulus response strength for several hue stimuli, e.g., differential imaging, might allow carving out a spatial organization that could not be shown here.

\section{Imaging color domains}

In all cases the strongest pattern formation was obtained with red/green flicker. In one case we also found clear response patterns to blue/yellow flicker. A second case showed faint pattern formation due to blue/yellow flicker. In that case we observed a general reduction in signal strength throughout the experiment. We can only assume that the anesthesia affected the intrinsic response in this case such that the cortical response to the blue/yellow flicker was weakened. In 2DG studies in macaques by Tootell et al. (1988) a similar preference for red/green and blue/yellow stimuli can be seen. However, they also show a faint blob pattern formation due to green/gray and yellow/gray stimuli. So far only one OI study (Xiao et al., 2007) known to us reported color selective responses in $\mathrm{V} 1$ due to green and yellow hue stimuli. Others failed to show reliable activation of blobs by stimuli other than red/green gratings (Landisman and Ts'o, 2002). This is surprising in light of anatomical studies (Sincich and Horton, 2005) showing that $\mathrm{KC}$ inputs target specifically blobs of the upper layers $2 / 3$, suggesting that $\mathrm{S}$-cone-selective stimuli should allow demarcation of blobs more effectively than $(\mathrm{L}-\mathrm{M})$-cone-specific stimuli that activate the PC-pathway, which provides direct inputs to layer $4 \mathrm{C} \beta$ without restriction to blobs.

\section{Dichromats versus trichromats}

Buzás et al. (2008) specifically addressed the question whether $\mathrm{S}$-cone inputs are segregated within V1 of dichromatic marmosets by using an OI approach. Single-condition maps of the intrinsic response to red/green and blue/yellow gratings did not reveal any pattern. They concluded that $\mathrm{S}$-cone inputs are distrib- 

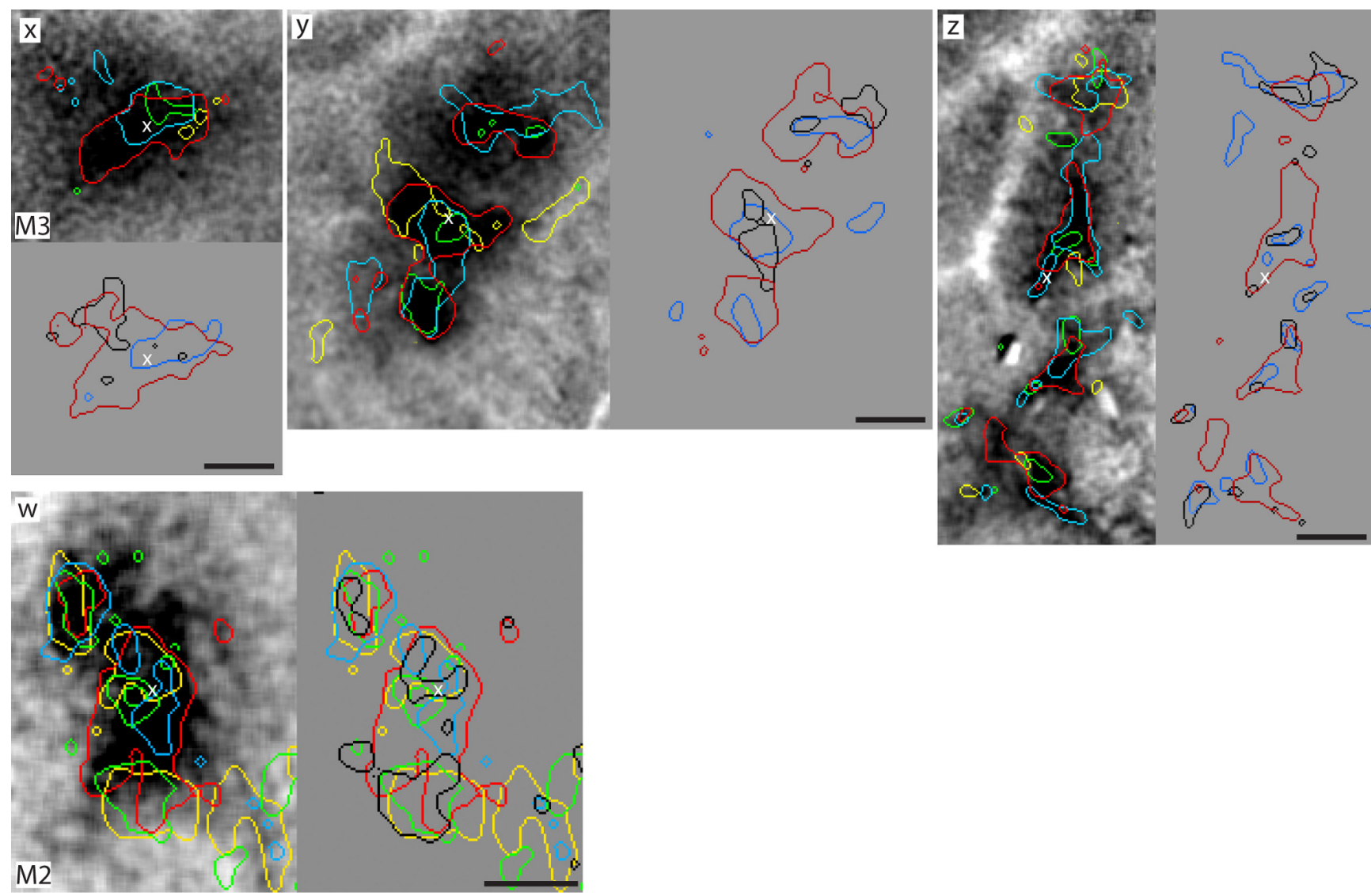

Figure 11. Spatial organization of color patches in V2. Contours of different color-varying stimuli from cases M2 and M3 are shown superimposed onto the patches in ROIs $(\boldsymbol{x}, \boldsymbol{y}, \boldsymbol{z})$ from Figure $10 \mathrm{G}$ for case M3, and Figure 5 (red/green) for case M2. Color code: bright red = red/gray, green = green/gray, yellow = yellow/gray, bright blue = blue/gray. Next to the ROls $\boldsymbol{x}, \boldsymbol{y}$, and $\boldsymbol{z}$, the contours of color-varying and of the luminance-varying stimulus responses are shown. Color code: dark blue $=$ blue/yellow, dark red $=$ red/green, black $=$ black/white. Scale bars $500 \mu \mathrm{m}$. For case M2 ( $\boldsymbol{w}$ ), color-varying stimulus responses are plotted together with luminance-varying stimulus responses (black contours).

uted homogeneously in marmosets, but acknowledged: “... we cannot rule out the possibility that there does exist a small subset of V1 cells with strong S-cone input. .. which we simply did not encounter" (p. 6).

All monkeys used in our study were shown to be genotypically trichromats. Although we did not confirm the phenotype by physiological recordings as done by Buzás et al. (2008), we found the typical pattern of color responses that is observed in macaques with red/green grating stimuli. The finding of color domains in our trichromats and their absence, in the work of Buzás et al. (2008), in dichromats suggests a dependence of trichromatism and functional blob activation. Our analysis of the patch-interpatch activity difference revealed that the only stimulus-dimension that accounted for a significant amount of variance was the $(\mathrm{L}-\mathrm{M})$-cone contrast axis, with a much reduced contribution of the $\mathrm{S}$ axis and no contribution of the ( $\mathrm{L}+\mathrm{M})$-axis (Fig. 8). This therefore confirms prior results in macaques, where patch formation is specific to $(\mathrm{L}-\mathrm{M})$-stimuli.

It is important to note that a variety of different stimulus protocols has been used throughout all corresponding OI studies. It is known that even isoluminant color contrast stimuli can activate color-blind orientation or motion selective $\mathrm{M}$ cells (Logothetis, 1991). In differential imaging approaches the response to an achromatic grating is subtracted from that to a red/green grating to isolate the faint color-specific response from orientation selective responses. This protocol proved to reliably reveal color patches in macaques (Landis- man and Ts'o, 2002; Lu and Roe, 2008). No study so far reported color patch patterns due to solitary red/green grating presentation. In preliminary experiments (data not shown here) we experienced that in some cases the intrinsic response to color opponent red/green gratings revealed faint patterns. When compared with response patterns from equally oriented achromatic gratings it emerged that these patterns were induced by the orientation of the chromatic grating. Thus, also differential maps calculated from those two conditions were dominated by the orientation response pattern. Neither the differential maps nor the single-condition maps yielded any indication for color domains. Thus, a singlecondition color grating approach as used by Buzás et al. (2008) or differential imaging as used in macaque studies might not be the appropriate stimulus protocols to reveal color domains in marmosets.

Solomon (2002) shows that the distribution of KC inputs within V1 does not differ in dichromatic and trichromatic marmosets thus supporting evidence from the macaque (Fitzpatrick et al., 1983; Horton, 1984; Hendry and Yoshioka, 1994) and owl monkey (Horton, 1984; Xu et al., 2004) that S-cone-selective inputs target specifically blobs (Solomon, 2002). Hence, it is not implausible that a blue/yellow flicker might reveal specific blob activation in trichromatic and also dichromatic marmosets. However, a specific blob activation by red/green flicker seems unlikely due to the lack of color opponent PC cells in dichromatic marmosets (Yeh et al., 1995). Interestingly, the peak response amplitudes of the ach- 
romatic and chromatic stimuli published by Buzás et al.(2008) show remarkably small differences (see their Table 1). In contrast, our results show that the response amplitudes of the achromatic gratings are around twice as high as the chromatic response amplitudes (Table 3). Buzás et al. (2008) report amplitude sizes of red/green gratings that differ by less than one third from the peak amplitudes of the achromatic gratings or even exceeds them. This suggests that a strong response to the orientation of the chromatic grating stimulus was induced that contributed to the color response and thus obliterated the faint color domains. Buzás et al. (2008) themselves state: “. . . we cannot rule out the possibility that the suppressive effect of large-field grating stimuli. . . has masked a weak but specific input from S-cone-carrying afferents to blob regions..." (p. 12). To dispel doubts we suggest that further OI studies on the color system of dichromatic marmosets should employ color opponent flicker stimuli. Such stimuli have proved to be a valuable tool for a specific blob activation in $\mathrm{V} 1$ of macaques (Tootell et al., 1988) and of marmosets (present study). In addition this approach allows to image color patches by a non-differential imaging approach, thus meeting the criteria for high resolution OI (Vanzetta et al., 2004). Finally, we conclude that the blob activation seen in our maps is primarily triggered by a modulation of the $(\mathrm{L}-\mathrm{M})$-cone axis, thus supporting the notion that blobs in New World as well as Old World trichromatic primates are domains of color processing.

\section{References}

Adams DL, Horton JC (2009) Ocular dominance columns: enigmas and challenges. Neuroscientist 15:62-77.

Benirschke K, Anderson JM, Brownhill LE (1962) Marrow chimerism in marmosets. Science 138:513-515.

Berwick J, Johnston D, Jones M, Martindale J, Redgrave P, McLoughlin N, Schiessl I, Mayhew JEW (2005) Neurovascular coupling investigated with two-dimensional optical imaging spectroscopy in rat whisker barrel cortex. Eur J Neurosci 22:1655-1666.

Blessing EM, Solomon SG, Hashemi-Nezhad M, Morris BJ, Martin PR (2004) Chromatic and spatial properties of parvocellular cells in the lateral geniculate nucleus of the marmoset (Callithrix jacchus). J Physiol 557:229-245.

Buzás P, Szmajda BA, Hashemi-Nezhad M, Dreher B, Martin PR (2008) Color signals in the primary visual cortex of marmosets. J Vis 8:7.1-7.16.

Casagrande VA (1994) A third parallel visual pathway to primate area V1. Trends Neurosci 17:305-310.

Conway BR (2009) Color vision, cones, and color-coding in the cortex. Neuroscientist 15:274-290.

Ding Y, Casagrande VA (1997) The distribution and morphology of LGN K pathway axons within the layers and CO blobs of owl monkey V1. Vis Neurosci 14:691-704.

Ding Y, Casagrande VA (1998) Synaptic and neurochemical characterization of parallel pathways to the cytochrome oxidase blobs of primate visual cortex. J Comp Neurol 391:429-443.

Fitzpatrick D, Itoh K, Diamond IT (1983) The laminar organization of the lateral geniculate body and the striate cortex in the squirrel monkey (Saimiri sciureus). J Neurosci 3:673-702.

Forte JD, Blessing EM, Buzás P, Martin PR (2006) Contribution of chromatic aberrations to color signals in the primate visual system. J Vis 6:97-105.

Hendry SH, Yoshioka T (1994) A neurochemically distinct third channel in the macaque dorsal lateral geniculate nucleus. Science 264:575-577.

Horton JC (1984) Cytochrome oxidase patches: a new cytoarchitectonic feature of monkey visual cortex. Philos Trans R Soc Lond B Biol Sci 304:199-253.

Horton JC, Hedley-Whyte ET (1984) Mapping of cytochrome oxidase patches and ocular dominance columns in human visual cortex. Philos Trans R Soc Lond B Biol Sci 304:255-272.

Horton JC, Hubel DH (1981) Regular patchy distribution of cytochrome oxidase staining in primary visual cortex of macaque monkey. Nature 292:762-764.
Hunt DM, Williams AJ, Bowmaker JK, Mollon JD (1993) Structure and evolution of the polymorphic photopigment gene of the marmoset. Vision Res 33:147-154.

Jacobs GH, Neitz J, Neitz M (1993) Genetic basis of polymorphism in the color vision of platyrrhine monkeys. Vision Res 33:269-274.

Kawamura S, Hirai M, Takenaka O, Radlwimmer FB, Yokoyama S (2001) Genomic and spectral analyses of long to middle wavelength-sensitive visual pigments of common marmoset (Callithrix jacchus). Gene 269:45-51.

Landisman CE, Ts'o DY (2002) Color processing in macaque striate cortex: relationships to ocular dominance, cytochrome oxidase, and orientation. J Neurophysiol 87:3126-3137.

Lim H, Wang Y, Xiao Y, Hu M, Felleman DJ (2009) Organization of hue selectivity in macaque V2 thin stripes. J Neurophysiol 102:2603-2615.

Livingstone MS, Hubel DH (1984) Anatomy and physiology of a color system in the primate visual cortex. J Neurosci 4:309-356.

Logothetis NK (1991) Is movement perception color blind? Curr Biol $1: 298-300$.

Lu HD, Roe AW (2007) Optical imaging of contrast response in macaque monkey V1 and V2. Cereb Cortex 17:2675-2695.

Lu HD, Roe AW (2008) Functional organization of color domains in V1 and V2 of macaque monkey revealed by optical imaging. Cereb Cortex 18:516-533.

Martin PR (2004) Colour through the thalamus. Clin Exp Optom $87: 249-257$.

Mayhew JEW, Askew S, Zheng Y, Porrill J, Westby GWM, Redgrave P, Rector DM, Harper RM (1996) Cerebral vasomotion: a 0.1-Hz oscillation in reflected light imaging of neural activity. Neuroimage 4:183-193.

Michael CR (1981) Columnar organization of color cells in monkey's striate cortex. J Neurophysiol 46:587-604.

Polimeni JR, Granquist-Fraser D, Wood RJ, Schwartz EL (2005) Physical limits to spatial resolution of optical recording: clarifying the spatial structure of cortical hypercolumns. Proc Natl Acad Sci USA 102:4158-4163.

Roe AW, Ts'o DY (1999) Specificity of color connectivity between primate V1 and V2. J Neurophysiol 82:2719-2730.

Roe AW, Lu HD, Hung CP (2005a) Cortical processing of a brightness illusion. Proc Natl Acad Sci U S A 102:3869-3874.

Roe AW, Fritsches K, Pettigrew JD (2005b) Optical imaging of functional organization of V1 and V2 in marmoset visual cortex. Anat Rec A Discov Mol Cell Evol Biol 287:1213-1225.

Schaeffel F, Hagel G, Eikermann J, Collett T (1994) Lower-field myopia and astigmatism in amphibians and chickens. J Opt Soc Am A 11:487-495.

Seymour K, Clifford CWG, Logothetis NK, Bartels A (2009) The coding of color, motion, and their conjunction in the human visual cortex. Curr Biol 19:177-183.

Sheinberg DL, Logothetis NK (2001) Noticing familiar objects in real world scenes: the role of temporal cortical neurons in natural vision. J Neurosci 21:1340-1350.

Shmuel A, Chaimow D, Raddatz G, Ugurbil K, Yacoub E (2010) Mechanisms underlying decoding at 7 T: ocular dominance columns, broad structures, and macroscopic blood vessels in V1 convey information on the stimulated eye. Neuroimage 49:1957-1964.

Signer E, Anzenberger G, Jeffreys A (2000) Chimaeric and constitutive DNA fingerprints in the common marmoset (Callithrix jacchus). Primates 41:49-61.

Sincich LC, Horton JC (2005) The circuitry of V1 and V2: integration of color, form, and motion. Annu Rev Neurosci 28:303-326.

Solomon SG (2002) Striate cortex in dichromatic and trichromatic marmosets: neurochemical compartmentalization and geniculate input. J Comp Neurol 450:366-381.

Spatz WB (1989) Loss of ocular dominance columns with maturity in the monkey, Callithrix jacchus. Brain Res 488:376-380.

Tootell RB, Hamilton SL (1989) Functional anatomy of the second visual area (V2) in the macaque. J Neurosci 9:2620-2644.

Tootell RB, Silverman MS, Hamilton SL, De Valois RL, Switkes E (1988) Functional anatomy of macaque striate cortex. III. Color. J Neurosci 8:1569-1593.

Tootell RB, Nelissen K, Vanduffel W, Orban GA (2004) Search for color 'center(s)' in macaque visual cortex. Cereb Cortex 14:353-363. 
Troilo D, Quinn N, Baker K (2007) Accommodation and induced myopia in marmosets. Vision Res 47:1228-1244.

Ts'o DY, Roe AW, Gilbert CD (2001) A hierarchy of the functional organization for color, form and disparity in primate visual area V2. Vision Res 41:1333-1349.

Valverde Salzmann MF, Wallace DJ, Logothetis NK, Schüz A (2011) Multimodal vessel mapping for precise large area alignment of functional optical imaging data to neuroanatomical preparations in marmosets. J Neurosci Methods 201:159-172.

Vanzetta I, Slovin H, Omer DB, Grinvald A (2004) Columnar resolution of blood volume and oximetry functional maps in the behaving monkey: implications for fMRI. Neuron 42:843-854.

Williams AJ, Hunt DM, Bowmaker JK, Mollon JD (1992) The polymorphic photopigments of the marmoset: spectral tuning and genetic basis. EMBO J 11:2039-2045.

Wong-Riley M (1979) Changes in the visual system of monocularly sutured or enucleated cats demonstrable with cytochrome oxidase histochemistry. Brain Res 171:11-28.

Xiao Y, Wang Y, Felleman DJ (2003) A spatially organized representation of colour in macaque cortical area V2. Nature 421:535-539.

Xiao Y, Casti A, Xiao J, Kaplan E (2007) Hue maps in primate striate cortex. Neuroimage 35:771-786.

Xu X, Bosking W, Sáry G, Stefansic J, Shima D, Casagrande V (2004) Functional organization of visual cortex in the owl monkey. J Neurosci 24:6237-6247.

Yeh T, Lee BB, Kremers J, Cowing JA, Hunt DM, Martin PR, Troy JB (1995) Visual responses in the lateral geniculate nucleus of dichromatic and trichromatic marmosets (Callithrix jacchus). J Neurosci 15:7892-7904.

Yoshioka T, Dow BM (1996) Color, orientation and cytochrome oxidase reactivity in areas V1, V2 and V4 of macaque monkey visual cortex. Behav Brain Res 76:71-88. 